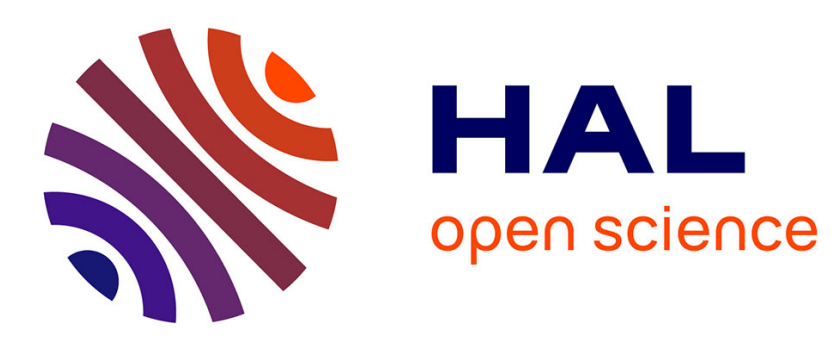

\title{
Double variation (auxiliaire et participe passé) au sein du parfait corse

\author{
Pierre-Don Giancarli
}

\section{To cite this version:}

Pierre-Don Giancarli. Double variation (auxiliaire et participe passé) au sein du parfait corse. Cahiers Chronos, 2016, Aoristes et parfaits en français, latin, corse, estonien et polonais, $\mathrm{n}^{\circ} 28$, pp.33-73. 10.1163/9789004313408_005. hal-01634221

\section{HAL Id: hal-01634221 \\ https://hal.science/hal-01634221}

Submitted on 14 Dec 2017

HAL is a multi-disciplinary open access archive for the deposit and dissemination of scientific research documents, whether they are published or not. The documents may come from teaching and research institutions in France or abroad, or from public or private research centers.
L'archive ouverte pluridisciplinaire HAL, est destinée au dépôt et à la diffusion de documents scientifiques de niveau recherche, publiés ou non, émanant des établissements d'enseignement et de recherche français ou étrangers, des laboratoires publics ou privés. 
GIANCARLI Pierre-Don, (2016), "Double variation (auxiliaire et participe passé) au sein du parfait corse", Cahiers Chronos, 28, Collection Language and Linguistics, Brill/Rodopi, P.-D. Giancarli \& M. Fryd (ed.), Aoristes et parfaits, En français, latin, corse, estonien et polonais, 33-73, Leiden/Boston

\begin{abstract}
In parallel with an aorist still in use (passatu landanu), Corsican has a periphrastic perfect (passatu quancianu) consisting of an auxiliary followed by a past participle. The auxiliary is variable since either avè (have) or esse (be) can be selected, primarily on the basis of thematic roles. The past participle is variable too since it alternates between a short form and a long one according to aspectual/modal criteria. This double variation obtains both within simple intransitive verbs and reflexive ones. Drawing on authentic extracts from Corsican corpora we shall take up the issue of this double variation in each of the domains concerned, before the question of a possible correlation between the two variations is addressed.
\end{abstract}

\title{
0. Introduction
}

Le corse est l'une des 14 langues romanes actuelles ${ }^{1}$. Il possède un aoriste, forme synthétique à valeur de passé appelée passatu landanu, et un parfait nommé passatu quancianu (mot à mot passé de ce côté-ci) qui est formé de l'association d'un auxiliaire conjugué au présent et d'ailleurs variable, et suivi d'un participe passé qui peut lui aussi varier.

Le passatu landanu est le temps de la rupture temporelle par rapport au moment d'énonciation repère $T_{O}$. C'est lui que, à côté du présent, l'on trouve dans les proverbes car, à partir d'une occurrence ancrée dans le révolu, le passatu landanu acquiert une valeur de vérité intemporelle et générale. Nous en donnons ci-dessous quelques exemples, assortis de leurs équivalents en français rendus au présent :

(1c) A pappula fatta da trè donni ùn si poti manghjà.

(1f) La tourte faite par trois femmes ne put jamais être mangée / Trois femmes ensemble bavardent trop pour bien faire la cuisine.

(2c) À rombu, à rombu a funa strappò.

(2f) À force à force la corde cassa / Tant va la cruche à l'eau qu'à la fin elle se casse.

Il s'emploie à l'écrit et à l'oral. A l'écrit, comme le montrent les extraits suivants. Ces traductions en corse d'exemples français au passé composé temporel sont éloquentes par leur non-choix de la forme auxiliée corse, dans des contextes narratifs ou avec datation temporelle :

(3f) Souvent, j'ai suivi des yeux les oiseaux de passage.

(3c) Seguitoiu à spessu cù 1'ochji l'acelli andarini chì mi vulavanu sopra. (G).

$1 \quad$ Lexicon der Romanischen Linguistik, 1988. 
(4f) Et la chambre est restée toute nue.

(4c) E a stanza firmó nuda nuda (LDM).

Et à l'oral en particulier chez ses locuteurs âgés ou instruits ${ }^{2}$, comme le prouvent les extraits ci-dessous. (5c) est une transcription d'enregistrement oral et (6c) est un passage dialogué de pièce de théâtre, autant d'aoristes en corse à mettre en parallèle avec leurs versions françaises au passé composé :

(5c) Arricurdatevine quella siratina induv'ì a tavula fù messa d'incantu...

(5f) Rappelez-vous cette soirée où la table a été mise par enchantement... (F).

(6c) - Arrimane ùn ci era micca ?

- Ma sì chi ci era. Ùn ti arracordi. Ancu un pocu è po ci ci appicchemu. Iè, hè cusì

(staglia e parolle) e-po-ci-ci a-ppicchemu. Ma tù ùn volsi.

(6f) - Il n'était pas là hier ?

- Mais si. Tu ne te rappelles pas. Il s'en est fallu d'un cheveu qu'on ne s'y soit pendu.

Oui, c'est juste (en détachant les mots) qu'on-ne-s'y-soit-pendu. Mais tu n'as pas voulu. (EAG).

Quant au parfait corse, comme l'écrit A. Culioli, il est utilisé dans le cadre suivant :

«le passé composé [le passé composé corse, c'est-à-dire pas le passé composé mais le passatu quancianu], que l'on trouve par exemple dans avali, aghju parlatu [= maintenant j'ai parlé] est utilisé lorsque l'action achevée a produit un résultat présent. [...] Le passé simple au contraire [le passé simple corse, c'est-à-dire le passatu landanu] est le temps de la narration. Alors qu'en français il est essentiellement utilisé dans la forme écrite, en corse on doit l'utiliser dans les discussions. Ainsi [pour dire l'autre jour il a mangé comme un cochon] dira-t-on l'altru ghjornu manghjò purcinettu et non hà manghjatu purcinettu. Mais, en s'adressant par exemple à un enfant pour le gronder on lui lancera en le prenant à témoin de sa propre saleté hai manghjatu purcinettu (Culioli et al.1998: 20)».

Cependant, l'aoriste corse connaît en ce moment et sous nos yeux une régression, corrélativement à une évolution du passatu quancianu et une redéfinition des rôles par laquelle le passatu quancianu peut devenir temporel, dépourvu d'état résultant et aussi inaccompli qu'une forme simple. Le tableau dressé par A. Culioli, s'il est valable pour des locuteurs âgés ou instruits, ne l'est plus pour tous les locuteurs, ce qui rend la situation mouvante et complexe.

A partir d'un corpus corse authentique d'environ 535.000 mots $^{3}$ et en recourant à quelques concepts de la Théorie des Opérations Prédicatives et Énonciatives (TOPE) d'A. Culioli, nous nous sommes penché sur la construction du parfait dans cette langue, en particulier sur sa double variation : variation d'auxiliaire (avoir ou être), et une seconde

2 Une grammaire d'aujourd'hui le décrit "Di registru di lingua cutidianu quant'è literariu (puru ch'ellu venga adupratu più pocu, oghje, da i lucutori ghjovani) [...]" (Franchi 2000 : 112) : de registre quotidien autant que littéraire (bien qu'il s'emploie moins aujourd'hui chez les jeunes).

3 En majorité en texte original mais incluant aussi quelques traductions officielles en langue corse à partir de textes français avec lesquels la comparaison peut permettre de mettre en relief la spécificité des choix du corse, comme pour les exemples 3 à 6 déjà donnés. 
variation qui touche le participe passé et que dans une première approximation on peut appeler long ou court.

Sachant que les deux variables se répartissent différemment : pour ce qui est des auxiliaires, au sein des intransitifs certains prennent seulement avè (avoir), d'autres seulement esse (être), et d'autres varient. Au sein des verbes pronominaux tous peuvent prendre esse, certains varient, mais aucun ne prend seulement avè. Pour ce qui est des participes, tous les verbes ont à disposition un participe long. Au sein des verbes en particulier bivalents certains peuvent varier entre participe long et participe court, mais aucun n'a seulement un participe court.

Nous aborderons d'abord la variation de participe, puis la variation d'auxiliaire, dans les deux domaines que sont les verbes simples et les verbes pronominaux, avant de nous poser la question d'une éventuelle corrélation entre ces deux variations.

\section{Variation de participe}

\subsection{De la nécessité de distinguer forme longue et forme courte}

Que les formes corses longue et courte diffèrent peut être montré au travers des quatre biais suivants :

\subsubsection{Accroissement par changement lexical}

Quand en corse on veut exprimer un accroissement, au sens de l'expression d'une quantité supérieure d'une qualité donnée, on peut le faire par le biais de deux termes gradables juxtaposés : il s'agit de deux termes situés sur un même gradient ${ }^{4}$, donc une même propriété, mais placés à des endroits différents sur ce gradient, si bien que l'un et l'autre sont identiques en qualité mais différents en termes de degrés, l'un jouant pour l'autre le rôle de haut degré. Dans ce cas, il y a deux contraintes : la première est que le terme intensif, c'est-à-dire porteur de la quantité supérieure, soit mentionné en deuxième ; la seconde est que ce deuxième terme, s'il dispose de deux allomorphes un long et l'autre court, soit obligatoirement une forme courte.

Considérons les verbes truncà (trancher, fracturer) et mirzà (pourrir, carier), auxquels sont associables respectivement truncatu / troncu et mirzatu / merzu :

(7c) Stanca tronca, Anna Maria, pinsava ch'ella ùn pudarìa mai più vultà ind'u so paese.

(7f) Morte de fatigue, Anna Maria pensait qu'elle ne pourrait jamais plus retourner dans son village. (UP)

(8c) Esse riccu merzu.

(8f) Etre un gros richard, être riche à ne savoir que faire de son argent.

7c signifie mot à mot fatiguée au point d'atteindre la rupture, fatiguée jusqu'à être dans l'état d'être brisé, $8 \mathrm{c}$ signifie riche au point d'être pourri, la richesse et le pourrissement étant vus

4 En première approximation un gradient est, dans la TOPE, une échelle orientée. Nous apportons quelques précisions sur son statut dans la partie 1.3. 
en corse comme une seule et même qualité. Dans ces constructions la forme longue serait totalement agrammaticale :

(7'c) Stanca *truncata...

(8'c) Riccu *mirzatu...

\subsubsection{Accroissement par répétition}

La répétition est une autre manière de rendre le haut degré, en se plaçant sur le gradient directement dans une orientation vers le centre attracteur (cf. Culioli 1986: 165 et notre partie 1.3.), sans repérage par rapport à une propriété intermédiaire. Considérons le verbe siccà (sécher), auquel sont associables siccatu et seccu:

(9c) Li paria di sente un parapiglia è tene spripusitatu, un strascinime arrighitu di carreghe, u parlacciume di i zitelli, e ghjastemme di u maestru è un cliccu seccu seccu chì li dicia chì a chjave girava ind'a sarratura.

(9f) Il entendait un vacarme assourdissant, un déplacement bruyant de chaises, le bavardage des enfants, les blasphèmes du maître et un déclic sec qui lui signalait que la clé tournait dans la serrure. (DS)

Cette construction, elle aussi, n'est licite qu'avec exclusivement la forme courte, en l'occurrence seccu seccu dans le sens figuré de très bref:

(9’c) Un cliccu *siccatu siccatu.

\subsubsection{Coordination}

En cas de coordination avec un adjectif, c'est encore la forme courte qui doit être employée, la longue est inacceptable. Considérons l'adjectif chjara (claire au féminin), et le verbe palisà (dénoncer, montrer) auquel sont associables palisatu et palesu :

(10c) A causa di l'omicidiu di u 1993 era dunque, per ellu, una cosa chjara è palesa.

(10f) Le mobile de l'assassinat de 1993 était donc, pour lui, parfaitement clair et évident. (ICB.)

(10’c) Chjara è *palisata serait mal formé.

\subsubsection{Adjectif composé}

Enfin pour former un adjectif composé construit sur une juxtaposition Nom + Adjectif, quand un choix existe entre forme courte et forme longue seule une forme courte est acceptable. Considérons les verbes truncà (trancher, fracturer) et muzzà (couper, mutiler), auxquels sont associables respectivement truncatu ou troncu et muzzatu ou mozzu :

(11) Il est coupé du bras (manchot) se dit hè braccitroncu et jamais *hè braccitruncatu.

(12) Il est tranché de la jambe (unijambiste) se dit hè ghjambimozzu et jamais *hè ghjambimuzzatu. 
On retiendra de la partie 1.1. que forme longue et forme courte ne sont pas assimilables l'une à l'autre. Et on peut préciser : elles ne sont pas assimilables l'une à l'autre parce que ce sont deux termes de natures différentes, à savoir que la forme courte correspond à un adjectif, alors que la forme longue n'est pas un adjectif (nous dirons qu'elle correspond à un participe). C'est pour cela que seule la forme courte peut se coordonner à un adjectif c'est-à-dire un terme de même nature, qu'elle peut jouer le rôle d'intensifieur après un adjectif, qu'elle peut se voir répétée pour exprimer le haut degré d'une qualité, et qu'elle peut être le deuxième composant au sein d'un composé adjectival.

Ces deux formes diffèrent donc à plusieurs niveaux, que nous résumons :

1) De par leur longueur bien sûr.

2) De par leur accentuation aussi : la longue est accentuée sur l'avant-dernière syllabe c'est-àdire sur la voyelle tonique de la terminaison, tandis que la courte est accentuée sur la syllabe précédente, sur le radical.

3) Parfois, et cela est lié au point précédent, elles diffèrent aussi de par leur prononciation : parce que l'accentuation change la voyelle qui sera accentuée, les formes longues sont sujettes à un phénomène dit de "svucalatura" (perte de voyelle), qui consiste en une fermeture de la voyelle qui n'est pas sous accent, d'où par exemple palesu / palisatu ou mozzu / muzzatu.

4) De par leur nature : la forme longue correspond à un participe passé, et la forme courte correspond à un adjectif, et le corse différencie bien le participe de l'adjectif 5 .

Si forme courte et forme longue diffèrent notamment parce l'une est un adjectif et l'autre un participe, en quoi plus précisément un adjectif et un participe passé diffèrent-ils et qu'estce que cela nous permet de préciser sur la relation entre les deux paradigmes en question au sein d'un parfait ?

\subsection{En quoi adjectif et participe passé diffèrent-ils ?}

Un adjectif donne une qualité attribuée à son support, il se contente d'énoncer à son sujet une propriété à un instant $\mathrm{t}$. Il ne met pas en jeu une quelconque problématique événementielle ni d'accès à cette qualité.

Un participe passé par contre, comme l'indique son appellation de " participe », participe de deux natures, en l'occurrence il tient à la fois de l'adjectif et du verbe. Il se distingue donc de l'adjectif par une composante verbale, et plus précisément événementielle. Un participe passé ne se contente pas de dire d'un objet qu'il est tel qu'il est, il le resitue dans une histoire dans laquelle il n'a pas toujours été ainsi ; il a précédemment connu un état différent avant de

5 Les langues romanes qui disposent de deux verbes pour rendre l'existence, comme l'espagnol et le portugais, différencient adjectif et participe de surcroît au travers de l'emploi de ser ou estar, différence qui peut respectivement remplacer ou s'ajouter à celle déjà marquée par le terme qui le suit : ainsi en portugais esta palido (il a perdu ses couleurs) contraste avec es palido (il est habituellement pâle), et en espagnol es despertado il est en train de passer à l'état d'éveil) contraste avec està despierto (il est en situation d'éveil, ou il s'agit de quelqu'un d'éveillé) (De Dardel : 85, Laurent : 358). 
connaître une mutation par laquelle il est devenu autre. Il prend donc en compte la phase d'accès à la qualité en cause.

Les formes courtes, adjectivales, interviennent cependant dans les constructions au parfait. Pour autant nous ne parlons pas, pour la forme courte employée dans un parfait, d'une forme « raccourcie » ou «tronquée » à partir de la longue, ni ne voyons de «manque » d'infixe ${ }^{6}$. C'est une forme de plein droit, un adjectif, mais qui à côté des participes peut se prêter à un emploi de participe quand il est employé au sein d'un parfait ${ }^{7}$, rappelant en cela les étapes de la constitution du parfait dans ses origines latines ${ }^{8}$.

Les formes courtes corses se prêtent d'autant plus facilement à un emploi de participes que la contraction de avè (avoir), contraction qui en corse signe le passage de verbe de possession à auxiliaire opérateur de repérage, et donc de structure bi-clausale à monoclausale, se trouve aussi en présence d'une forme courte au sein de ce qui ne peut être qu'une structure mono-clausale au parfait ${ }^{9}$ :

(13c) M'eti salvu !.

(13f) Vous m'avez sauvé ! (CS.)

M'eti salvu, variante contractée avec forme courte de m'aveti salvu, ne signifie pas vous $m$ 'avez en tant que je suis sauf même si salvu aurait pu en tant qu'adjectif et à la différence de la forme longue salvatu se prêter à une telle interprétation, mais est incontestablement un parfait.

On se trouve donc en présence de paires minimales au parfait, avec forme longue ou forme courte, telles que, en traduction mot à mot, là où le français dit j'ai séché mon pantalon et j'ai lassé mon auditoire le corse peut dire soit j'ai séché mon pantalon soit j'ai sec mon pantalon et soit $j$ 'ai lassé mon auditoire soit $j$ 'ai las mon auditoire, et là où l'anglais dit $I$ have opened the door et I have awoken him le corse peut dire soit I have opened the door soit I have open the door et soit I have awoken him soit I have awake him.

Précisons que cette illustration par le biais du français ou de l'anglais a cependant ses limites, elle biaise même partiellement la réalité justement parce qu'en français séché / lassé

6 De Dardel (77-86) parle de « participes romans sans suffixe » et Durand (251) de participes «privi del formante $-V t u »$.

7 Rohlfs (378), parlant de certaines régions du Sud de la péninsule italienne, reconnaît au parfait la présence d'adjectifs pour assumer une fonction participiale. Cela serait fréquent avec un adjectif comme pieno au sens de rempli (son équivalent est inusité en corse), et il en trouve aussi des traces dans des textes anciens, notamment du début du $16^{\mathrm{e}}$ siècle.

8 En effet les participes passés latins sont originellement des adjectifs (Blatt : 158, Vincent : 81), et, dans les structures latines en habere à l'origine des parfaits romans, l'attribut de l'objet qui deviendra un participe passé est incontestablement un adjectif, comme le montre sa capacité à admettre le degré comparatif ou superlatif, construction typique non des verbes mais des adjectifs (Salvi 1982 : 120, et aussi Salvi 1987 : 227).

9 Les contractions sont possibles sur l'auxiliaire avè conjugué au présent à partir des formes trisyllabiques, et réduites alors à des dissyllabiques dépourvues de la première syllabe inaccentuée. Cette contraction concerne l'auxiliaire avè, mais pas le verbe avè, qui a une forme toujours pleine, c'est-à-dire que la réduction phonétique du signifiant doit refléter la subduction du signifié. 
ou en anglais opened / awoken sont des participes passés, et sec / las ou open / awake sont seulement des adjectifs qui ne peuvent pas, dans ces langues, prendre la place d'un participe. Or en corse cette double possibilité existe. Nous soutiendrons que, même au sein d'un parfait, les formes courtes gardent quelque chose de leur nature adjectivale, permettant ainsi de distinguer les emplois de parfait à forme longue, des emplois de parfait à forme courte (que nous désignerons dorénavant par parfait à forme longue et parfait à forme courte, ou de façon abrégée parfait long et parfait court).

\subsection{Forme longue et forme courte au sein d'un parfait}

La co-existence de ces deux formes permet des paires minimales de parfait telles que les suivantes, dont les membres ne sont pas tout à fait synonymes, même hors-contexte :

(14f) J'ai préparé le repas.

(14c1) Aghju acconciu a cena / (14c2) aghju accunciatu a cena.

(15f) J'ai chargé trois paquets sur le bateau.

(15c1) Aghju carcu trè pacchetta nant'à u batteddu / (15c2) aghju carcatu trè pacchetta nant'à u batteddu.

(16f) Pierre a taché sa chemise.

(16c1) Petru hà taccu a so camisgia / (16c2) Petru hà taccatu a so camisgia.

(17f) Pierre a taillé deux arbres.

(17c1) Petru hà mozzu dui arburi / (17c2) Petru hà muzzatu dui arburi.

La première version de $14 \mathrm{c}$ dit que c'est préparé, ou que ça a été préparé rapidement. La deuxième accentue l'idée de durée de la préparation.

La première version de $15 \mathrm{c}$ s'intéresse au fait que les paquets sont bel et bien chargés. La deuxième emporte l'idée de pénibilité de la tâche, ou laisse entendre qu'il y a d'autres paquets à charger.

La première version de $16 \mathrm{c}$ dit que c'est fait. La deuxième suggère que c'est un événement de plus, qui s'ajoute à d'autres.

La première version de $17 \mathrm{c}$ s'intéresse au fait que les deux arbres dont il est question sont bel et bien coupés, la glose pourrait en être voilà, c'est fait. La deuxième précise que cela n'a pas été sans mal, ou favorise la lecture selon laquelle il y a encore du travail à faire et des arbres à couper ${ }^{10}$.

Comment rendre compte de ces intuitions de façon théorique, tout en tenant compte de la nature fondamentale des termes en présence ?

Nous aurons besoin pour la suite du concept de Frontière, et donc de domaine notionnel, que nous empruntons à la TOPE et que nous présentons brièvement. A partir de la notion,

10 Concernant en particulier $15 \mathrm{c}$ à $17 \mathrm{c}$ dans leur version longue on ne peut s'empêcher de penser au fait qu'en latin, qui a connu le phénomène des doubles participes (cantum / cantatum, oblitum / oblitatum, votum / votatum, etc., voir conclusion), la forme longue est donnée comme ayant un sens itératif (De Dardel : 80-81, Laurent : 80). 
« système complexe de représentation structurant des propriétés physico-culturelles d'ordre cognitif » (Culioli 1982: 8), on construit un domaine notionnel. Ce domaine se compose de plusieurs zones : un extérieur E (qui incarne l'altérité), et son complémentaire linguistique l'intérieur I (valeur positive) qui comprend lui-même un centre organisateur, possédant toutes les caractéristiques de la notion en question, et un centre attracteur, occurrence possédant les propriétés définitoires de la notion à un degré élevé. Au centre attracteur est associé un gradient (évoqué brièvement dans la partie 1.1.), qui n'indique pas la présence de bornes et permet une orientation dans les deux sens : soit on se déplace vers le centre attracteur et on a alors de plus en plus la propriété en question, soit on s'en éloigne et on l'a alors de moins en moins. La Frontière F est un composant du domaine notionnel qui sépare E de I. Sans être ni I ni E, elle possède à la fois les propriétés de I et de E : c'est un intervalle, une valeur intermédiaire « qui a la propriété "p" et en même temps la propriété altérée qui fait que ce n'est plus totalement "p" sans que cela soit totalement extérieur» (Culioli 1986: 165). Ce n'est plus E, mais ce n'est pas encore I.

Dans ce cadre, redéfinissons ce que sont un adjectif et un participe passé :

Un participe passé repose sur une quantification, il renvoie en soi à la construction d'un domaine notionnel zoné, construit un changement d'état par franchissement de F, et place l'Intérieur I (par exemple palisatu ou muzzatu) en relation avec son complémentaire $\mathrm{E}$ « autre que palisatu ou muzzatu, autre que I ». Il construit un état qui est un état final (état2) dont il dit qu'il a été précédé d'un état antérieur (état1), après passage par la Frontière.

Un adjectif par contre ne renvoie pas en soi à la construction d'un domaine notionnel, il énonce une qualité et exprime l'état d'un argument.

Quand il est immergé au sein d'un parfait construit avec une forme courte, l'état incarné par l'adjectif sera, par la force des choses, un état2 c'est-à-dire un état résultant, mais il n'est pas soumis aux aléas de l'entrée dans une zone après transition depuis son complémentaire : le parfait à forme courte (palesu ou mozzu), à base d'adjectif, présente l'état résultant stabilisé comme forcément et pleinement atteint. On est pleinement en I, et le franchissement d'une Frontière sans épaisseur relève de la simple formalité.

Le parfait à forme longue (palisatu ou muzzatu) par contre, marque, avant atteinte d'un état résultant, le passage par une Frontière dilatée, avec sur-ajout de possibles valeurs modales.

Comment ceci se manifeste-t-il dans les textes, et en contexte car l'environnement discursif sera différent. Prenons le verbe cuntà (conter), auquel sont associables cuntatu ou contu, avec d'ailleurs un phénomène de svucalatura déjà évoqué. C'est la forme longue qui a été choisie dans le premier extrait, la courte dans le deuxième, les deux par le même auteur :

(18c) Aghju cuntatu i fatti, aghju dettu i nomi è rammintatu 1'epica.

(18f) J'ai raconté les faits, j'ai dit les noms et rappelé l'époque. (ABM)

On pourrait croire qu'il y a, entre Aghju cuntatu i fatti et les propositions énumérées à la suite (aghju dettu i nomi è rammintatu l'epica), une relation de consécutivité qui ferait que l'ordre d'apparition des verbes représenterait directement l'ordre chronologique des événements extralinguistiques. Nous ne le pensons pas car les propositions autres que la première sont 
comprises dans la première. On pourrait d'ailleurs imaginer une ponctuation explicitante avec les deux caractéristiques suivantes :

- d'une part des points de suspension, car l'énumération des objets racontés continue, et se termine d'ailleurs elle-même par des points de suspension indiquant une liste ouverte ${ }^{11}$.

- et d'autre part une ponctuation sous forme de parenthèses ou de tirets, encadrant tout ce qui suit la première proposition afin de baliser les groupes et d'expliciter les niveaux de structuration, montrant qu'il s'agit du développement de composantes recouvertes par le premier GN objet du premier verbe :

(18'c) Aghju cuntatu i fatti (i nomi, 1'epica...).

(18'f) J'ai raconté les faits (les noms, l'époque...).

Les procès qui suivent cuntà (c'est-à-dire dì / dire et rammintà / rappeler) sont des verbes de parole qui sont autant de gloses intralingues de cuntà, si bien qu'on pourrait s'en tenir à ce seul verbe cuntà pris comme dénominateur commun, et auquel sont également à rapporter les objets des verbes dì et rammintà, aussi bien i nomi, l'epica que a Madonna, a barca, $e$ madonne di tutti i rughjoni. U portu novu. A Piazza cù e madonne. L'odore di a cera è di u calore. E vampate di e rosule, i rivocchi di ghjente, u sussurru in ogni locu. I cunfratelli è l'abiti. Incamisgiati sin'à l'ochji. E mantellette turchine...

Les procès d'après développent le premier procès cuntà, dont ils sont autant de sousintervalles, procès cuntà qui, au travers de ces synonymes, s'allonge ainsi indéfiniment avant d'en arriver à son terme à savoir la quantification du dernier objet de son dernier sousintervalle, puisque chaque objet constitue une quantification de cuntà. Ces sous-intervalles ont pour effet de reculer le moment où l'on va enfin déboucher sur un état résultant. On n'y arrive même jamais vraiment, dans la mesure où la liste des objets est donnée comme liste ouverte, incomplète. On peut en effet toujours ajouter un dernier point. Du à raconter reste à faire, le raconté n'est donc pas totalement atteint et réalisé. Le sujet peut certes s'arrêter de raconter, il peut interrompre son activité d'énumération, mais cela ne signifie pas qu'il l'aura achevée. Il ne sera pas pour autant allé à son terme vu que la totalité de l'objet n'a pas été affectée et n'est pas passée de E à I.

Par contre, avec le même verbe et par le même auteur, on trouve la forme courte (contu) dans :

(19c) Niculaiu mi purtava unu annu o dui, à u ghjustu ùn sò più. Ma l'età impreme pocu. $\mathrm{A}$ causa hè di più u rispettu ch'avia per quelli ch'o cunsiderava i nostri capi. Vi aghju contu digià quella di u sgaiullu turnatu impiegatu giardinaru à a merria è prumessu, in a mo giuventù, à a carriera di un capitanu di guerra. E' bè, à Niculaiu u tenia cum'è à quessu, è credu ch'o u temia ancu una cria.

11 Voici la suite de l'extrait, que nous donnons seulement dans sa traduction en français pour raison de place et qui est fidèle dans ce domaine au texte corse de départ, composé de GN coordonnés ou juxtaposés, tous objets du verbe de parole, avec des points de suspension en finale : Et puis la Madone, la barque, les madones de toutes les régions. Le nouveau port. La Place, avec les madones. L'odeur de la cire et de la chaleur. Les bouffées de roses, les flots de monde, l'agitation en tout lieu. Les confrères et les aubes. Couverts jusqu'aux yeux. Les mantelets bleus... 
(19f) Nicolas avait un an ou deux de plus que moi, je ne sais plus au juste. Mais l'âge importe moins que le respect que j'avais pour ceux que je considérais comme nos chefs. Je vous ai déjà raconté l'épisode du garnement devenu par la suite jardinier municipal et qui était promis dans mon enfance à la carrière d'un capitaine de guerre. Eh bien, je respectais Nicolas autant que celui-là, et je crois bien que je le craignais un peu. (AFA)

Ici l'énonciateur fait un constat, un bilan à $T_{O}$ d'un procès qui a eu lieu antérieurement, considère le révolu pour parler du résultat que le révolu a laissé dans l'actuel à savoir l'incontestable connaissance que ses interlocuteurs sont censés avoir de l'histoire du garnement promu employé de la Mairie.

L'adverbe digià (déjà) montre qu'on est d'emblée dans l'intérieur I, dans le validé. L'atteinte de I est faite, et elle est même confirmée. Il s'agit d'un rappel à l'interlocuteur de quelque chose qu'il sait au moment d'énonciation. A partir de cette connaissance partagée dont est réactivée la pertinence dans le discours présent, et qui joue le rôle de nécessaire repère dans une comparaison d'égalité impliquant Niculaiu en fin de séquence, l'énonciateur peut enchaîner sur Niculaiu en le reprenant comme thème. Il fallait que le positionnement en I soit acquis et incontestable pour que, dans une étape suivante avec laquelle la transition est marquée par E' bè (Eh bien), la référenciation de la phrase d'après avec quessu (celui-là) soit interprétable (à Niculaiu u tenia cum'è à quessu / Nicolas je le respectais autant que celui-là). D'où l'importance d'une certaine cohérence discursive dans le choix de la forme longue ou courte.

L'exemple suivant nous permettra de montrer comment s'effectue le passage de valeurs aspectuelles à des valeurs modales. Considérons le verbe circà (chercher, rechercher), auquel sont associables circatu ou cercu. La forme longue a été choisie en (20) :

(20c) È quandu u populu veni pruibitu, a so cultura pudarà essa altru cà una contru à cultura ? È a so storia, edda, pudarà essa altru cà una contru à storia ? S'eddu si vulia trattena, u populu, è mantenasi intrevu, li vinia ubligata à resista. È a so storia, da u principiu ad $a v a ̀$, hè propiu impastata d'una risistenza pirmanenti à i forzi di fora, ma ancu à quiddi di drentu chì ani circatu di rompa, à longu andà, u bilanciu paru di tutti quanti i cumpunenti di u populu corsu.

(20f) Quand le peuple se voit interdit, sa culture pourrait-elle être autre chose qu'une contreculture ? Son histoire, autre chose qu'une contre-histoire ? S'il entendait se poursuivre, le peuple, et se maintenir intact, il se voyait contraint de résister. Depuis l'origine et jusqu'à ce jour, son histoire est proprement pétrie d'une résistance permanente aux forces extérieures mais également à celles de l'intérieur qui ont cherché à rompre, à la longue, le juste équilibre de toutes les composantes du peuple corse. (IE)

D'un point de vue aspectuel, le parfait à forme longue concourt, on l'a vu, à allonger la phase de transition en repoussant la borne de droite de l'intervalle, dilate l'intervalle et encode grammaticalement que cette tentative de rompre prend du temps. Ici cela va de pair avec le laps de temps évoqué par le Groupe Prépositionnel circonstant dans le contexte-gauche da $u$ principiu ad avà / depuis l'origine jusqu'à aujourd'hui.

Mais cette tentative (jugée néfaste par l'énonciateur) de rompre la résistance et la cohésion du peuple corse prend du temps aussi pour la raison qu'elle ne va pas sans rencontrer une vive résistance de la part de l'agressé ( $E$ a so storia [...] hè propiu impastata d'una risistenza pirmanenti / son histoire est proprement pétrie d'une résistance permanente), 
elle se heurte à une intentionnalité adverse et se solde même par un échec, dans un passage qui est globalement un hymne à ce que perdurent la résistance et l'équilibre du peuple corse.

Avec les verbes marqueurs d'une opération de visée ${ }^{12}$ comme circà (il en irait exactement de même avec pruvà / essayer), c'est non seulement circà mais aussi sa complémentation verbale qu'il faut prendre en considération : l'énonciateur distingue une des deux valeurs complémentaires du domaine notionnel, en l'occurrence I, puisque la forme positive a été sélectionnée. Un objectif, ayant pour source des forces jugées perturbatrices et auquel l'énonciateur n'adhère pas, est posé comme à atteindre (cet objectif est rompa $u$ bilanciu / rompre l'équilibre), et une distance est posée comme à combler si cet objectif doit être atteint.

Le choix d'un parfait à forme longue ou d'un parfait à forme courte permet de préciser (d'anticiper sur) le sort qui sera celui de cette distance, comblée ou non-comblée, si le validable va être transformé en validé ou en non-validé, et donc s'il en résultera succès ou échec de la tentative.

En l'occurrence avec un parfait long, il y a préconstruction de l'échec de visée du prédicat (rompa u bilanciu). Le référent du sujet grammatical vise un objectif (mettons I), mais en même temps l'énonciateur fait savoir/prévient que c'est le chemin menant au complémentaire (E) qui sera le cas. Il y a, par rapport à la valeur qui est visée, une orientation inverse du préconstruit. Avec un parfait long, l'énonciateur privilégie grammaticalement la non-validation de la relation prédicative.

Par contre avec le même verbe et par le même auteur, la forme courte est choisie en (21) :

(21c) Aghju cercu di cunsultà u dussariu ghjudiziali di Martinu Appinzapalu. Aghju ottinutu a dìruga cassinnò bisogna aspittà 100 anni !.

(21f) J'ai cherché à consulter le dossier judiciaire de Martinu Appinzapalu. J'ai obtenu la dérogation car sinon il faut attendre 100 ans ! (UT) ${ }^{13}$

Avec un parfait court il y a préconstruction de validation du prédicat (cunsultà...). Le référent du sujet grammatical vise un objectif (en l'occurrence I), et l'énonciateur fait savoir que le chemin menant à cet objectif (l'Intérieur I) sera effectivement le cas. Il y a, par rapport à la valeur visée, une orientation à l'identique du préconstruit. Et c'est bien d'ailleurs ce que précise le contexte-droit, puisqu'il y a réussite du projet entrepris : Aghju ottinutu a diruga / J'ai obtenu la dérogation.

Résumons les résultats auxquels nous sommes parvenus dans cette partie 1.3. : alors que, grâce à une Frontière sans épaisseur, la forme courte dit simplement que p est le cas ${ }^{14}$, la

12 Dans la TOPE la visée est une relation de rupture (relation aoristique) entre l'énonciation de la visée (repère énonciatif à partir duquel on vise la validation) et la situation pour laquelle la relation prédicative sera éventuellement validée (Culioli 1990 : 146-147) mais n'est en réalité que validable.

13 Martinu Appinzapalu a écrit notamment dans la revue corse $A$ Muvra, tentée par les idées d'extrême droite de l'irrédentisme mussolinien. Accusé de menacer l'intégrité nationale, il fut jeté en prison après la Libération, où il mourut d'un cancer.

14 Exemples 14c1 Aghju acconciu a cena, 15c1 aghju carcu trè pacchetta nant'à u batteddu, $16 \mathrm{c} 1$ hà taccu a so camisgia, 17, 1 hà mozzu dui arburi, 19 Vi aghju contu digià quella $[\ldots]$. 
forme longue, marqueur d'une Frontière avec épaisseur (intervalle dilaté), emporte les indications aspectuelles / modales / aspectuo-modales que

- c'est le cas MAIS cela a pris du temps ${ }^{15}$, ou est une occurrence médiane précédée ${ }^{16}$ ou appelée à être suivie ${ }^{17} \mathrm{~d}$ 'autres occurrences donnant de l'épaisseur à l'intervalle

- c'est le cas MAIS difficilement ${ }^{18}$

- c'est le cas MAIS ça ne devrait pas l'être ${ }^{19}$

- c'est le cas MAIS seulement de façon temporaire, il y a instabilité de l'état résultant ${ }^{20}$

- c'est peut-être le cas MAIS il s'agit moins d'une assertion que d'une hypothèse cantonnée au domaine du non-certain ${ }^{21}$.

Et, avec des verbes de visée, se mettent en place des préconstruits orientés à l'inverse l'un de l'autre : la forme courte privilégie et garantit l'atteinte de validation (21 aghju cercu di [...]), tandis que la forme longue ne permet pas de déboucher sur l'occurrence visée, il y a échec de validation (20 ani circatu di [...]).

Que c'est à sa composante participiale que le parfait est redevable de ces interprétations se trouve en outre corroboré par le fait que, même en dehors de la forme finie qu'est le parfait, le participe employé seul, dans des constructions appelées « absolues », continue à mettre en œuvre les invariants mis au jour. Considérons dans l'extrait suivant le verbe tuccà (toucher / impacter en tant que transitif), qui permet tиссаtu ou toсcu :

(24c) A libartà, a porta l'omu addossu, in a parsona è in a so menti, com'è una surgenti buddiccinanti di vita ancu à sbramà. Secca à tempu à l'omu, è vivi a tempu ad eddu, in u daziu ch'eddu faci sempri vulinteri in pratesa di locu. Tиссаtu ad unu, tocси u populu tuttu quantu.

(24f) La liberté, l'homme la porte en lui, dans sa personne et dans son esprit, comme une source bouillonnante de vie et non encore venue à satiété. Elle s'assèche en même temps

15 Exemples 14c2 Aghju accunciatu a cena et 18 Aghju cuntatu i fatti, aghju dettu i nomi è rammintatu l'epica.

Exemple 16c2 Hà taccatu a so camisgia.

17 Exemples 15c2 Aghju carcatu trè pacchetta nant'à u batteddu et 17c2 Hà muzzatu dui arburi.

18 Exemples 15c2 Aghju carcatu trè pacchetta nant'à u batteddu et 17c2 Hà muzzatu dui arburi.

Exemple 20 Ani circatu di [...].

20 (22c) Ciò ch'ellu c'è, in prima, in lu puema, è a Pruvenza - a Pruvenza di u mare, a Pruvenza di a muntagna - cu a so storia, e so legende, $i$ so paisaggi, tuttu u so populu niscentre e libaru chi ha truvatu u so gran pueta prima di sparisce ... / (22f) Ce qu'il y a avant tout dans le poème, c'est la Provence, - la Provence de la mer, la Provence de la montagne, - avec son histoire, ses moeurs, ses légendes, ses paysages, tout un peuple naïf et libre qui a trouvé son grand poète avant de mourir... (LDM).

21 (23c) Trà elle è caccaru hè cume s'elli avianu qualcosa ch'elli spartenu inseme elli soli. Soca serà ch'elli si sò avvizzati tremindui à issa solita passighjata. / (23f) Entre grandpère et lui c'est comme s'ils avaient, eux seuls, quelque chose en commun. C'est (le corse dit ce sera au futur épistémique pour renforcer l'hypothèse) peut-être qu'ils ont pris goût tous les deux à cette promenade régulière. (ABM). 
que l'homme et elle vit en même temps que lui, dans le don qu'il fait toujours volontiers en défense du lieu. Un seul être est touché et tout le peuple l'est. (IE)

Il s'agit dans Tиссати ad ипи toсси и рориlu de deux propositions participiales p et q en construction absolue et donc en relation parataxique, $\mathrm{p}$ jouant le rôle de repère et en l'occurrence de cause, q jouant le rôle de conséquence et d'état résultant. P comporte un participe long (tuccatu), et q un participe court (toccu), et ce pour le même verbe.

Cette première différence formelle est accompagnée d'une seconde : la forme longue (en p) est munie d'un accusatif prépositionnel ${ }^{22}$, tandis que la forme courte (en q) est sans accusatif prépositionnel. Cette double différence est d'autant plus remarquable au sein d'une structure habituellement fortement empreinte de symétrie. L'accusatif prépositionnel devant l'objet nous permet de déduire 1) que ипи est objet et pas sujet, 2) que le sujet est donc autre que ипи (en l'occurrence il s'agit d'un agent X non-instancié et générique) et 3) que tuccatu est de nature verbale et plus précisément un participe passé actif, permettant la glose : $X$ ha tиссаtи (ad) ипи. Unи est un terme sémantiquement patient en fonction objet dans une relation active, et pas un terme sémantiquement patient en fonction sujet dans une relation passive.

L'accusatif prépositionnel montre aussi que l'objet se protège de l'affectation que la transitivité est censée lui imposer, et la validation n'est pas assurée : si pour $\mathrm{p}$ on reconstruit une proposition subordonnée tensée ce serait davantage si quelqu'un a touché à [...] que quand quelqu'un a touché à [...], avec une relation d'entraînement unidirectionnelle entre $\mathrm{p}$ et q, relation d'entraînement qu'on peut gloser de la façon suivante au parfait :

(24c') Si calchissia hà tuccatu ad unu di noi, hè com'è ch'eddu hà toccu u populu sanu.

(24f') Si quelqu'un a touché un seul d'entre nous, c'est comme s'il a touché la totalité du peuple.

La proposition $p$ est validable mais pas validée, par contre si $p$ est validée alors l'énonciateur garantit qu'en q il y aura résultat atteint, d'où également le choix de la forme courte. La prise en charge éventuelle de p revient au sujet non-instancié X, et celle de q et du lien (quasiment causal) de $\mathrm{p}$ à q revient à l'énonciateur. En d'autres termes, si la validation de p par l'agent non-instancié X se fait (et elle est déconseillée par l'énonciateur, comme le montre toute la phrase et la prise en compte du segment q en particulier, et en p l'accusatif prépositionnel et le participe à forme longue, puisque la non-validation est le chemin préférentiel), alors c'est l'atteinte de la validation de q qui est garantie par l'énonciateur, et on peut se situer d'emblée en I à l'aide d'une forme courte. Il s'agit donc globalement d'une mise en garde.

C'est une des raisons pour lesquelles nous ne parlons pas de pluractionnel : il est vrai que cette situation du corse, comme nous le suggère pertinemment notre collègue Gerhard Schaden (Université de Lille 3), pourrait laisser penser au pluractionnel. Cela est d'autant plus tentant que cette dimension a déjà été mise en évidence avec un parfait, celui du portugais en ter aussi bien au Portugal (Amaral \& Howe 2013) que dans une partie du Brésil (CabredoHofherr, Laca \& Carvalho 2010).

Rappelons que le terme de «pluractionnel » fut au départ utilisé en linguistique africaine pour désigner toute forme verbale indiquant une pluralité d'actions / sujets / objets, et donc

22 Sur l'accusatif prépositionnel en corse voir Giancarli 2014. 
fondée sur l'itération (Newman 2012 : 185). À la pluralité de procès c'est-à-dire l'itératif s'est parfois ajouté le duratif (Gerhardt 1984 in Newman, idem). Peut-on, dans cette vision déjà large du pluractionnel, intégrer le parfait corse dans sa version longue ?

Dans la mesure où ce dernier ne se cantonne pas à l'itératif / duratif mais permet une dimension modale, nous pensons difficile de réduire l'infixe -AT- à un marqueur pluractionnel. De plus, alors que l'effet pluractionnel du parfait portugais jugé unique dans les langues romanes serait imputable à son auxiliaire ter (issu de tenere et pas de habere comme il l'est en corse) (Giorgi \& Pianesi 1997, Cabredo-Hofherr et al. 2010 : 95), nous arrivons à notre interprétation aspectuo-modale du parfait corse dans sa version longue en nous appuyant sur son participe, et pas sur son auxiliaire. Nous l'avons démontré notamment ci-dessus par le fait que, même en dehors du parfait, le participe employé sans auxiliaire dans les constructions absolues continue à mettre en œuvre les invariants mis au jour.

Voilà pour l'exposé de la première variation, qui concernait le participe. Abordons à présent la seconde.

\section{Variation d'auxiliaire}

Concernant la seconde variation au sein du parfait, celle non pas de participe passé mais d'auxiliaire, nous nous appuierons sur certaines conclusions de travaux antérieurs (Giancarli 2011 et 2015) avant de nous poser la question d'un éventuel lien entre les deux types de variation. Nous aurons besoin de distinguer entre verbes simples et verbes pronominaux.

\subsection{Verbes simples}

Dans le cadre d'une vision scalaire des macro-rôles inspirée de la Role and Reference Grammar de W. Foley \& R. Van Valin, nous appelons Source et But non pas des notions pourvues de rôles thématiques tel agent et patient mais des macro-rôles thématiques assignables à des notions (appelées à devenir des arguments) et englobant les rôles d'agent et de patient.

Et nous soutenons que l'auxiliaire avè (avoir) est sélectionné quand le sujet possède le macro-rôle de terme Source, avec des propriétés primitives agissantes, et esse (être) quand le sujet correspond à la fois à un terme Source et un terme But, instigateur agissant et affecté subissant, et assume donc deux rôles thématiques. Cette idée fondamentale vaut pour la sélection de esse à la fois avec les verbes simples, qui seront exclusivement des monovalents, et avec les pronominaux, qui peuvent être monovalents ou bivalents. Mais alors que pour les premiers le sujet possède ce double statut de façon intrinsèque, avec les seconds ce double statut est le plus souvent de façon acquis par le biais d'une co-référence.

Chaque macro-rôle se décline en plusieurs rôles organisés en une hiérarchie : agent volitif, agent non-volitif, instrument et stimulus pour le premier. Patient, bénéficiaire, destinataire, thème, siège-expérient et siège locatif pour le deuxième. Nous laissons de côté deux éléments qui jouent un rôle en corse dans la sélection d'auxiliaire pour lesquels nous renvoyons à Giancarli 2015, et qui relèvent de la syntaxe et de la servilité.

Examinons quelques verbes simples monovalents admettant une variation. Au sein des verbes de processus correspondant à ces critères se trouvent ceux qui sélectionnent esse quand 
le sujet grammatical est Source et But inanimé, ou bien Source et But animé mais non volitif, ou encore Source et But animé mais non contrôleur.

1) Sujet Source et But animé et agentif (avè) / sujet Source et But inanimé (esse)

Parce que le sujet d'un verbe à auxiliaire essif ${ }^{23}$ comporte une dimension subissante, on trouve avec de tels verbes une répartition simple selon laquelle le verbe est essif quand son sujet est exclusivement inanimé (et donc évidemment inagentif), ce qui rend la composante But prépondérante, et par complémentarité c'est avè qui est employé quand le sujet est animé et agentif, ce qui rend la composante Source prépondérante et la composante But insuffisamment perceptible. D'où le contraste ci-dessous entre (25) et (26) avec vulà (voler) :

(25c) Bulà, aghju bulatu un pocu inghjilocu è a giograffia, hè virità, m'hè ghjuvata assai.

(25f) J'ai volé un peu partout dans le monde. Et la géographie, c'est exact, m'a beaucoup servi. (PP)

(26c) Hè vulatu à pezzi.

(26f) Il a volé en éclats. (Ceccaldi : 443)

Vulà prend avè en (25) avec un sujet animé de première personne et a alors son sens propre, ici voler dans les airs à bord d'un avion, mais il se construit avec esse quand son sujet est inanimé. Il prend alors un sens figuré, en (26) voler en éclats, mot à mot il est volé à morceaux. Même répartition dans les extraits ci-dessous avec saltà (sauter) :

(27c) Dopu d'avè saltatu da a ghjumenta, Michelu capu calatu, a voce dolce è ferma, disse :

(27f) Après avoir mis pied à terre, Michelu d'une voix douce et ferme dit : (UP)

(28c) Sò agrancata, Lucia hè stata tomba quand'hè saltata a posta d'Alger.

(28f) Je suis tétanisée... Lucie a été tuée dans l'attentat de la Poste d'Alger... (LT)

En (27) le sujet récupérable pour saltà est animé (Michelu) et avè est sélectionné, en (28) qui signifie mot à mot Lucie a été tuée quand elle est sautée la poste d'Alger le même verbe est conjugué par esse car le sujet est inanimé (la poste d'Alger).

2) Sujet Source et But animé et volitif (avè) / sujet Source et But animé mais non volitif (esse)

Nous prenons l'exemple de cambià (changer). Ce verbe suit souvent la répartition avè + sujet animé / esse + sujet inanimé, mais il permet de mettre au jour une nuance supplémentaire au sein des animés. Comparons les exemples (29) et (30) :

(29c) Vidi o Marcu, com'iddu faci, ùn hà micca cambiatu, sempri impressu à u soldu.

(29f) Tu vois, Marco, ses manières, il a pas changé, toujours derrière le fric. (PAV)

(30c) Sete pò cambiatu, sapete !

(30f) Comme vous avez changé ! (EAG)

En (30c), mot à mot Oh oui que vous êtes changé vous savez !, cambià se conjugue avec esse bien que son sujet soit animé, parce que son sujet subit un changement involontaire, avec le

23 Essif renvoie à l'auxiliaire esse, et à l'auxiliaire être de façon interlingue. 
temps. Avè par contre est choisi en (29) dans ùn hà micca cambiatu car le délocuté a choisi de ne pas faire ce qui lui aurait permis de changer, il a choisi de ne pas changer de la façon jugée pourtant utile par l'énonciateur et de persister dans son comportement vénal. Au sein des sujets animés, avè sera donc choisi pour indiquer que le sujet animé est volitif, et en esse qu'il ne l'est pas forcément.

3) Sujet animé Source et But partiellement contrôleur (AVOIR) / sujet animé Source et But mais non contrôleur (ÊTRE)

Les verbes sguillì (glisser) et sculiscià (glisser avec mouvement descendant) illustrent une variation proche des précédentes. On aurait pu se contenter de dire que sguillì et sculiscià vont de pair avec esse quand le sujet est inanimé, et avec avè quand il est animé. Mais la répartition est plus subtile. Esse est sélectionné avec les inanimés car ils sont entièrement inagentifs :

(31c) [les gâteaux dans une boîte posée sur les genoux glissent à l'intérieur de la boîte] I vecu chì sò sguilliti drentu à u cartone è si sò accatamansati in 1'angulu di a scatula, da a mo parte.

(31f) Je vois qu'ils ont glissé dans le carton et se sont entassés dans l'angle de la boîte, de mon côté. (ICB)

Mais au sein des animés on peut trouver l'un ou l'autre auxiliaire, aussi bien le avè attendu et qui figure effectivement en (33), que esse, comme le montre (32) :

(32c) Ma, vulendu schisà u pede d'un muraglione, s'hè abbicinatu troppu, è sguillitu, e chi sà cum'ellu s'hè sbrugliatu, s'è trovu à anche in sù, e di capu ind' una ceppa di prunalbellu.

(32f) Mais en voulant éviter le bas d'un grand mur, il s'est trop approché de la planche inférieure, il a glissé et qui sait comment il s'y est pris, il s'est retrouvé les jambes en l'air, la tête dans un buisson d'aubépines. (CEC)

(33c) Hà sguillitu nantu à a neve è s'hè campatu.

(33f) Il a fait de la glisse sur la neige et il s'est régalé.

En (32) esse se justifie par le fait que la glissade du référent du sujet s'est soldée par une chute (chute évidemment accidentelle c'est-à-dire non-volitive, en l'occurrence il s'est retrouvé les jambes en l'air, la tête dans un buisson d'aubépines), il y a eu chute car le sujet est dépourvu de contrôle sur le procès. Le sujet est Source + But avec renforcement de la composante But.

Par contre en (33) avec avè, le procès est totalement volitif et désiré, comme le montre la compatibilité avec $s$ 'hè campatu / il s'est régalé, auquel cas le sens de ce verbe d'activité n'est pas « glisser par accident » mais « faire une glissade » (ou plus naturellement «faire des glissades », fà sguillate), par exemple sur un toboggan, ou «faire de la glisse » / « faire du patinage » si l'activité se pratique sur de la glace. Avè s'emploie donc avec un sujet Source et But qui est contrôleur, esse avec un sujet Source et But mais qui est non contrôleur.

Notre explication s'applique aussi, mutatis mutandis, à la sélection d'auxiliaire au sein de la forme pronominale (truncassi / se casser, buscassi / s'attraper, etc.), comme nous allons le montrer. 


\subsection{Verbes pronominaux}

Nous voyons en $s i$, le clitique pronominal, un argument. Ou plus exactement un quasiargument car c'est un argument partiellement déficient ${ }^{24}$. Plus précisément nous faisons de $s i$ un quasi-argument terme Source (argument externe) co-référent au sujet, sujet qui se retrouve de ce fait doté d'un double statut. C'est parce que si est déjà terme Source que l'énoncé dans lequel il figure n'admet pas l'expression d'un complément d'agent (Source), même quand la version pronominale semble sémantiquement proche d'un passif, alors que le passif l'admet :

(34c) E ghjembe sò state tronche / (34c') e ghjembe sò state tronche da Petru.

(34f) Les branches ont été cassées / (34f') les branches ont été cassées par Pierre.

(35c) E ghjembe si sò tronche / (35c') *e ghjembe si sò tronche da Petru.

(35f) Les branches se sont cassées / (35f') *les branches se sont cassées par Pierre.

Le sujet grammatical est à priori terme But, mais pas seulement But. Il n'est pas seulement But parce que si et le sujet grammatical entrent en relation de co-référence. C'est parce que si est terme Source et qu'il est en relation de co-référence avec le sujet que le sujet n'est pas seulement terme But, d'où notre troisième point :

Nous faisons de si un (quasi-)argument terme Source mais pas pour autant du sujet un argument terme But car le sujet acquiert le rôle thématique complémentaire c'est-à-dire Source par le biais d'une co-référence avec si et acquiert donc un double statut But + Source.

C'est ce double statut qui est la raison de la sélection de l'auxiliaire esse pour le pronominal. Si du moins deux autres critères, les critères de valence et d'orientation ne viennent pas modifier ce choix, car le pronominal corse admet en réalité les deux auxiliaires, esse ou avè. Plus précisément certains types de pronominaux sont ouverts à variation, qu'il nous faut à présent préciser.

\subsubsection{Valence}

Nous distinguons 11 emplois au sein du pronominal corse, qui tous permettent esse. Parmi eux 4 permettent non seulement esse mais aussi avè, autrement dit une variation d'auxiliaire, les $n^{\circ} 2,3 a, 4 b$ et $8 b$ :

24 En tant que clitique il connaît des contraintes, en particulier de placement et d'accentuation. Il est en outre limité à la fonction sujet, cf. Giancarli 2011. 


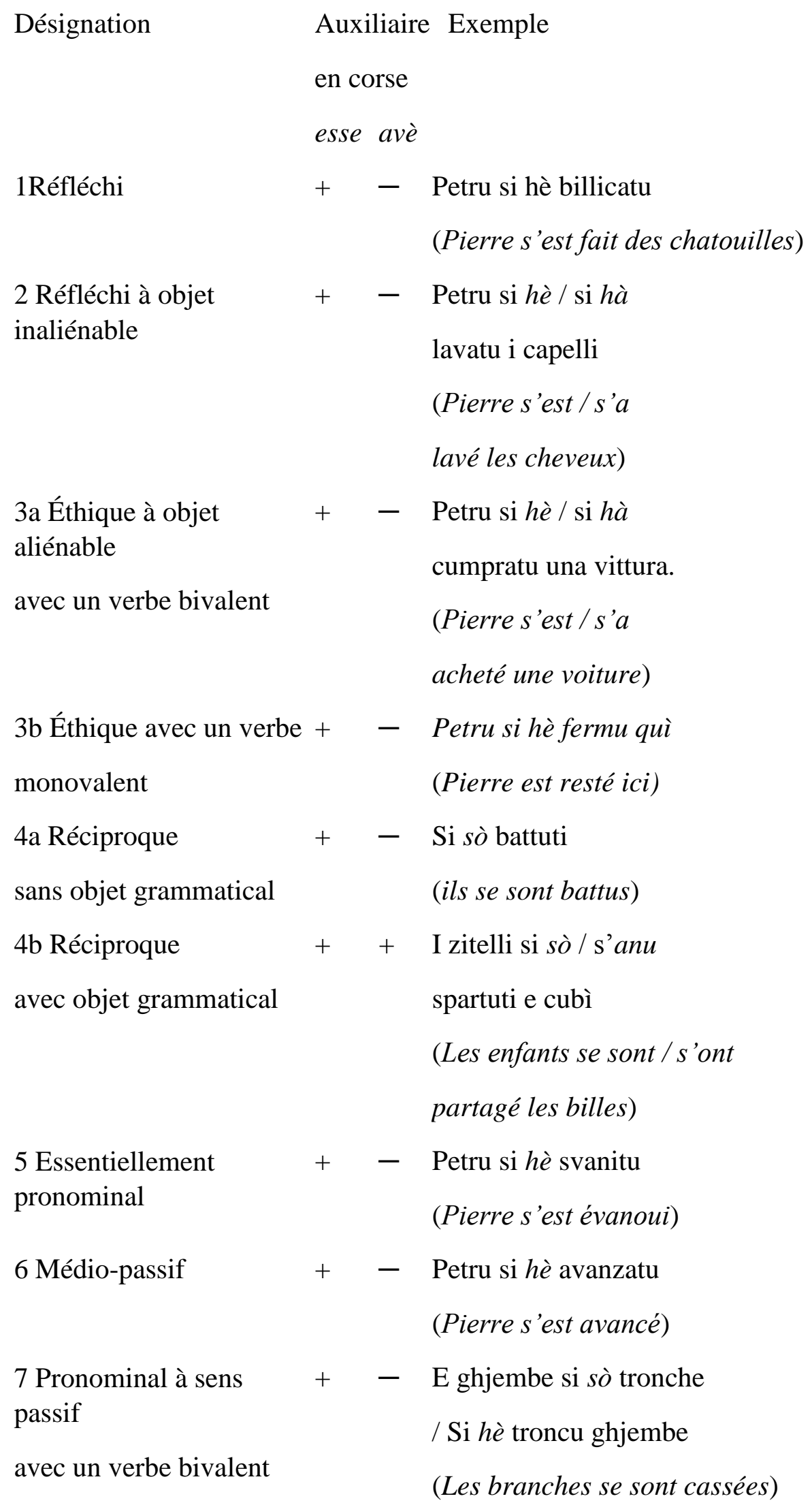




\begin{tabular}{|c|c|c|c|}
\hline $\begin{array}{l}\text { 8a Pronominal avec un } \\
\text { verbe monovalent } \\
\text { inaccusatif }\end{array}$ & + & - & $\begin{array}{l}\text { Si hè andati } \\
\text { (On (y) est allés) }\end{array}$ \\
\hline $\begin{array}{l}\text { 8b Pronominal avec un } \\
\text { verbe monovalent }\end{array}$ & + & + & $\begin{array}{l}\text { Si hè / si hà travagliatu } \\
\text { (On est travaillé / on a travaillé) }\end{array}$ \\
\hline
\end{tabular}

inergatif

Tableau 1 : Les emplois du pronominal corse en relation avec l'auxiliaire.

C'est sur ces quatre emplois, qui permettent une variation, que nous nous concentrerons. Qu'ont en commun les formes pronominales ouvertes à avè ? S'il n'y avait pas $8 \mathrm{~b}$, la réponse serait clairement : la présence d'un verbe bivalent et la présence d'un objet grammatical. Cela est net quand on compare 1-3b-4a-5-6-7, où tout passage à l'auxiliaire avè est bloqué, à respectivement 2-3a-4b-8b, qui permettent avè. Mais il y a $8 b$.

Essayons cependant de prendre en compte $8 \mathrm{~b}$, illustrable par si hè /hà travagliatu (on a travaillé) ou si hè/hà parlatu (on a parlé). Bien qu'on n'ait là ni verbe bivalent ni objet grammatical, mais des verbes monovalents, on peut ramener ce type d'exemple (monovalents inergatifs) au fonctionnement des formes bivalentes 2-3a-4b, et ce par le biais de l'objet interne (cf. par exemple Hale \& Keyser 2002) :

À la différence des inaccusatifs, clairement monovalents, les inergatifs « globalisent un procès en intégrant les spécifications qui pourraient être exprimées par des compléments » (cf. Riegel et al. : 221). Herslund 1996 est encore plus radical et va jusqu'à dire que les inergatifs ne sont pas de vrais monovalents, mais des bivalents déguisés en monovalents. Ils ont en réalité un objet, mais au lieu que cet objet soit externalisé sous la forme d'un GN après le verbe il est lexicalisé à l'intérieur de la racine verbale, ce qu'on désigne commodément sous le nom d'objet interne. On peut ainsi gloser voyager par faire un voyage ou jouer par faire un jeu. Nous en dirons autant de travaglià, parlà (parler), girivoltulà (pirouetter), ortulà (jardiner), guerrighjà (guerroyer), etc. : travaglià signifie faire du travail c'est-à-dire produire du travail par le fait de travailler, parlà signifie émettre des paroles par le fait de parler. On a d'ailleurs parfois des équivalences entre verbes monovalents inergatifs et verbes formellement bivalents munis d'un objet, ce qui tend à montrer que ces pseudo-monovalents intègrent bien un objet : girivoltulà est glosable par fà a girivolta tout comme pirouetter par faire des pirouettes, ortulà est glosable par fà / piantà l'ortu tout comme jardiner par faire son jardin, etc. Et cet objet est bien un argument, comme le montre le test des causatives ${ }^{25}$.

Ce rapprochement des monovalents inergatifs avec les bivalents est une manière de justifier la possible sélection de avè avec les inergatifs de $8 \mathrm{~b}$. Nous dirons donc que la série qui comporte un objet est aussi celle qui permet l'auxiliaire avè. La sélection de avè implique

25 Avec un verbe bivalent le corse introduit son objet indirect par une préposition quand il y a déjà un argument complément d'objet direct : Petru face fà a girivolta à Paulu (Pierre fait faire des pirouettes à Paul) implique en raison de à Paulu que a girivolta est un argument en fonction objet. 
obligatoirement la présence d'un objet, la série qui n'a pas d'objet ne permet jamais avè, et doit en rester à esse. Le choix d'auxiliaire a donc clairement quelque chose à voir avec la valence.

Cependant s'il est exact que la sélection de avè implique obligatoirement la présence d'un objet, la présence d'un objet n'implique pas la sélection de avè : en effet, l'ajout d'un argument objet rend l'auxiliaire avè possible ${ }^{26}$ mais pas contraint puisque esse reste sélectionnable ${ }^{27}$. Le critère de la valence est donc au mieux partiellement pertinent pour ce qui est du choix de l'auxiliaire dans les constructions pronominales en corse. Nous sommes face à une nouvelle variation d'auxiliaire, mais à nombre d'arguments égal, donc dans un rapport qui n'est que lâche avec la valence. Il est nécessaire pour comprendre l'alternance des auxiliaires dans le pronominal corse de continuer vers une explication complémentaire, à savoir l'orientation.

\subsubsection{Orientation}

Nous prenons orientation au sens de mise en topique ${ }^{28}$, et dirons que l'énonciateur sélectionne avè (dans les constructions bivalentes) pour manifester une orientation vers l'objet présent, et esse pour une orientation-sujet en dépit de la présence d'un objet. Nous donnerons à titre d'illustration de cette variation une paire d'exemples qui sont des pronominaux réciproques avec objet, sur le même verbe, en l'occurrence sparte (partager), conjugué avec esse en (36) et avè en (37) :

(36c) Microcosimu u paese ? Ben intesu, a dritta è a manca, i neri è i rossi, dui partiti chì si sò spartuti una cintunara d'elettori, cuntendu i braganati.

(36f) Le village, est-il un microcosme ? Bien entendu, la droite et la gauche, les noirs et les rouges, deux tendances qui se sont partagé une centaine d'électeurs, en comptant bien sûr ceux qui étaient « caméléon ». (DF)

(37c) - Alora, spartaremmu i vacchi, avali !

- Seti vo! Seti vo!

S'hani spartutu i vacchi, dici alora : Qualissi voli ?

(37f) - Alors, nous partagerons les vaches, maintenant!

- Comme vous voulez! Comme vous voulez! Lesquelles veux-tu ? (CS)

En (36) sparte est associé à l'auxiliaire esse qui permet de valoriser les sujets destinataires, les électeurs n'étant que quantité négligeable, des jouets manipulés permettant aux élus de devenir des notables. Avè (dui partiti chì s'anu spartutu una cintunara d'elettori) aurait

26 Cf. le pronominal réfléchi, tel Petru si hà lavatu $i$ capelli, le pronominal éthique à objet aliénable, tel Petru si hà cumpratu una vittura, le pronominal réciproque, tel $i$ zitelli s'anu spartutu e cubì, le pronominal à sens passif inergatif, tel si hà travagliatu.

27 Cf. respectivement Petru si hè lavatu i capelli, Petru si hè cumpratu una vittura, i zitelli si sò spartuti e cubì, si hè travagliatu.

28 Le topic (thème) s'oppose au rhème (comment, commentaire, ce qui est dit de nouveau sur le thème) et se définit en complémentarité avec lui. Le rhème se définit par la réponse qu'il apporterait à une éventuelle question, le thème se définit comme ce dont on parle, ce dont il est question, ce dont l'énoncé dit quelque chose, en contexte. 
donné à l'objet elettori un statut autonome échappant au contrôle du sujet, inadéquat dans le contexte insulaire.

En (37) trois frères, dont un est sensé et les deux autres un peu ballots, ont du mal à partager entre eux de façon équitable car le plus malin a déjà escroqué ses frères sur le partage du blé et du vin. Après ces deux objets, on passe à présent à un troisième objet soumis à partage, et d'autant plus central dans le débat que les deux frères escroqués ont décidé de ne plus se laisser berner. I vacchi dans s'hani spartutu $i$ vacchi (non-traduit dans la version française et signifiant mot à mot ils s'ont partagés les vaches) constitue l'élément sur lequel s'oriente le discours, et qui est aussi le support de la question Qualissi voli ? / Lesquelles veux-tu? Le choix de esse aurait centré sur le sujet, sujet collectif composé des trois frères au sein duquel aucune dissension concernant l'objet ne serait envisagée, et qui procèderaient d'un commun accord, dans le consensus voire la satisfaction, sans faire des vaches un enjeu.

Nous avons proposé des réponses à la question de la variation de participe et à la question de la variation d'auxiliaire en corse. Abordons à présent celle d'une éventuelle corrélation entre les deux variations.

\section{Recherche d'une corrélation entre les deux variations}

Sachant que le parfait corse connaît une double variation, variation d'auxiliaire (esse ou avè), et variation de participe (long ou court), on peut se poser la question de savoir s'il existe un lien entre ces deux variations, c'est-à-dire s'il y a des associations privilégiées entre tel auxiliaire et tel participe.

Rares sont les grammaires corses qui se positionnent sur ce point, ou qui fournissent même des exemples un tant soit peu indicatifs. L'une cependant, celle de J.-J. Franchi (2000), suggère par ses illustrations l'existence d'associations privilégiées, dans le domaine des verbes simples (piove / pleuvoir) comme dans celui des pronominaux (truncassi / se casser, pinsassi / se penser) :

Il a plu. Hà piuvitu / hè piossu (Franchi : 103). Il s'est cassé la jambe.

Si hà truncatu una ghjamba / si hè troncu una ghjamba (idem : 102).

On constate en effet dans ces exemples que les auxiliaires alternent de pair avec les participes. Et toujours de la même manière, la sélection de l'auxiliaire esse s'associant au choix du participe court et celle de avè avec le long. Et le lien entre les deux variations est même explicitement établi puisque l'ouvrage précise que c'est l'usage de la forme courte ou longue qui entraîne un changement d'auxiliaire (Franchi : 110).

Pour nous faire une idée plus précise de la question, nous voudrions, à partir de corpus, essayer d'en dresser une sorte de cartographie, dans le domaine des verbes simples puis dans celui des verbes pronominaux. 


\subsection{Verbes simples}

Il convient, au sein des verbes simples conjugués au parfait, de distinguer un certain nombre de cas :

a) Il y a les verbes qui ne varient ni leur participe ni leur auxiliaire car ils prennent seulement avè. Ceci concerne une majorité de verbes transitifs (par exemple dì / dire), et un très grand nombre d'intransitifs inergatifs (par exemple ortulà / jardiner).

b) Il y a ceux qui ne varient ni leur participe ni leur auxiliaire car ils prennent seulement esse. Ceci concerne certains intransitifs parmi ce que d'aucuns qualifieraient d'inaccusatifs et qui sont selon nous des verbes à double statut (cf. notre partie 2), qu'il s'agisse de verbes d'état (bastà / être suffisant) ou d'activité (sfilà / défiler).

c) Il y a ceux qui varient leur auxiliaire mais pas leur participe, nous en avons vu des exemples comme saltà / sauter, vulà / voler, cambià / changer et sguillì / glisser en 2.1.

d) Inversement il y a ceux qui, comme firmà / rester, varient leur participe (firmà permet firmatu ou fermu) mais pas leur auxiliaire car ils ne prennent que esse, pour la même raison que celle mentionnée en b).

e) Il y a ceux qui varient leur participe mais pas leur auxiliaire car ils ne prennent que avè, nous en avons vu des exemples en 1.3., tels accuncià / préparer, carcà / charger, circà / chercher, cuntà / conter ou muzzà / couper.

Tous ces verbes ne nous intéressent pas ici car ils ne répondent pas au critère d'une variation double.

Enfin, il y a f) les verbes simples qui varient à la fois leur auxiliaire et leur participe. Et à ce stade notre analyse sur corpus nous livre une première surprise, à savoir que nous n'avons relevé que trois verbes, qu'il convient sans doute de réduire à deux verbes, voire à un seul. Les trois candidats sont piove (pleuvoir), le verbe qui est cité dans la grammaire de Franchi ; cumincià (commencer) ; et turnà, ou turrà avec rhotacisme, plus polysémique.

En ce qui concerne piove, il admet bien deux participes, un long (piuvitu) et le sigmatique court piossu. Nous avons effectivement trouvé des occurrences de esse + participe court et des occurrences de avè + participe long conformément à l'association posée par Franchi, mais il y a aussi esse avec du long et avè avec du court. Outre un nombre d'exemples trop faible pour pouvoir se prêter à des statistiques pertinentes, ils se répartissent de façon quasi-homogène sur tous les choix possibles, si bien qu'aucune préférence ne se dégage.

Et, de toutes façons, la variation d'auxiliaire de ce verbe est à considérer avec prudence car la totalité des quelques emplois en avè relevés se limite à un seul auteur, M. Biancarelli, par ailleurs très ouvert à l'influence du français ${ }^{29}$. Sachant que le corse est en situation de

29 C'est aussi un des rares auteurs à donner un participe passé au verbe duvè (devoir) correspondant au français $d \hat{u}$, et à se permettre des formes hybrides avec le verbe essif stà comme ùn li so micca statu à senta / je ne les ai pas écoutés dans le verbe complexe stà à 
diglossie par rapport au français, nous faisons pour ce verbe et chez cet auteur l'hypothèse d'une influence exogène du français sur le corse, et considérons donc que piove n'est pas à comptabiliser parmi les verbes admettant de façon authentique une double variation, à la fois du participe et de l'auxiliaire, car hors influence du français ce verbe ne sélectionne que esse, comme d'ailleurs les autres verbes météorologiques ${ }^{30}$.

En ce qui concerne turnà, il admet effectivement deux participes passés, turnatu et tornu (ou avec rhotacisme turratu et torru). Et cette fois il est vrai que l'auxiliaire varie aussi. Du moins dans certains des sens que peut prendre turnà : pas en tant qu'intransitif au sens de devenir parce qu'alors il n'admet que esse; pas non plus en tant qu'intransitif au sens de revenir, pour la même raison; pas davantage en tant que transitif au sens de rentrer (ou faire rentrer) parce qu'alors il n'admet que avè. Mais dans des emplois tels que les suivants, au sens aspectuel de recommencer:

(41c) Stargone (hà turnatu ad attundà à Lucca).

(41f) Estragon (qui s'est remis à tourner autour de Lucky). (EAG)

(42c) Si sente u trustu d'una cascata. Dimiriu face i minguli par di ch'elli sò turnati à cascà.

(42f) Un bruit de chute, appuyé par la mimique de Vladimir, annonce qu'ils sont tombés à nouveau. (EAG)

Pour autant nous pensons que cette alternance d'auxiliaire relève d'une illusion : nous considérons en effet que ce verbe est, parallèlement à pudè (pouvoir) / vulè (vouloir, etc.) / duvè (devoir), un verbe servile. Une des particularités de ces verbes est d'être transparents : ils peuvent être précédés de l'un ou de l'autre auxiliaire, ce qui compte est le verbe qui a été choisi et l'auxiliaire qui aurait été le sien si le verbe avait été conjugué directement. Par exemple on a les deux auxiliaires en succession avec pudè verbe servile dans :

(43c) Avendu l'occasione di rivede la trenta anni dopu, Albert ùn hè pussutu stà ! Ùn hà pussutu risiste à a tentazione di ride si di sti pullami culuriti chì ornanu u paese di isti ribelliperusempre.

(43f) Ayant eu l'occasion de le revoir 30 ans après, Albert n'a pas pu se retenir ! Il n'a pas pu résister à la tentation de s'amuser à nouveau autour de ces animaux qui entourent le village des irréductibles. (RG)

Dans hè pussutu stà, le participe pussutu est précédé de esse parce que stà sélectionne esse, dans hà pussutu risiste par contre pussutu est précédé de avè parce que resiste sélectionne avè.

senta / écouter car une telle construction comprend à la fois l'auxiliaire esse, qui fait de stà un verbe libre mais omet de faire porter le clitique sur l'infinitif, et un clitique antéposé, qui fait de stà une forme liée mais omet de conjuguer le verbe complexe avec l'auxiliaire dans ce cas attendu.

30 Piuvicinà (bruiner, pleuviner), bambacinà (bruiner), diluvià (pleuvoir très fort), scandilà (faire des gouttes), candilleghjà (commencer à pleuvoir), nivà/nevicà (neiger), nivacciulà (neigeotter), fiuccà (tomber, pour neige ou pluie), gragnà (neiger à flocons à demi fondus), grandinà (grêler), vintulà (venter), brinà (givrer), ghillà (geler), incutrà (geler, glacer), sunità (tonner), tunà (tonner), tunizà (tonner au loin), lusinà (faire des éclairs), accindità (faire des éclairs lumineux), fulminà (faire des éclairs violents), etc. 
De même nous dirons qu'une variation a lieu devant turnà, mais pas en fonction de turnà. Elle se fait en fonction du verbe qui le suit : en 41 dans hà turnatu ad attundà à Lucca, où il s'agit bien ici du sens itératif de turnà (qui ne correspond donc pas à tourner dans le texte français mais à se remettre (à)/recommencer), le verbe est attundà (tourner), verbe simple incapable de sélectionner esse puisque bivalent, et qui entraîne le choix de avè. On dit hà turnatu ad attundà à Lucca comme on dirait hà attundatu à Lucca et pas *hè attundatu à Lucca. En 42 dans sò turnati à cascà le verbe qui suit est cascà (tomber), conjugué par esse. On dit sò turnati à cascà comme on dirait so cascati et pas *hanu cascatu.

Le verbe turnà, même dans son sens aspectuel de recommencer, n'admet donc pas une variation de l'auxiliaire puisque celle-ci est due non à lui mais au verbe qui le jouxte. Il n'admet donc pas une double variation, à la fois du participe et de l'auxiliaire, et ne se prête pas à l'examen d'une éventuelle corrélation entre elles.

Reste cumincià. Ce verbe permet bien une variation de son participe (cuminciatu ou cumenciu). Et il permet effectivement une variation d'auxiliaire dans la mesure où, comme corre, saltà, vulà ou principià, il sélectionne avè quand son sujet est Source et But animé et agentif, et esse soit quand son sujet est Source et But inanimé, soit quand son sujet est Source et But animé mais subissant, cf. partie 2 sur «Variation d'auxiliaire ».

Le corpus met-il en évidence des préférences de esse pour la forme courte, et de avè pour la forme longue ? Oui et non : il est vrai que le parfait long se trouve plus souvent avec avè (18 occurrences) qu'avec esse (5 occurrences), mais en revanche aucune association privilégiée n'apparaît entre esse et le parfait court ( 0 occurrence) comparé à avè avec parfait court (1 occurrence). Rien de très probant donc.

À supposer même qu'on comptabilise le verbe cumincià, ce qui nous paraît devoir être mis en évidence est qu'avec les verbes simples l'hypothèse d'une association de la sélection de l'auxiliaire esse avec un participe court et celle de avè avec un long est fragile sur cumincià, et surtout que le cas de figure d'une double variation est exceptionnel puisqu'il se réduit au mieux à un seul verbe, cumincià, qui est un hapax : la question d'une association au parfait entre tel auxiliaire et tel participe est une question qui ne se pose quasiment pas.

Du moins dans le domaine des verbes simples. Mais il y a aussi les verbes pronominaux, qu'il nous faut à présent aborder.

\subsection{Verbes pronominaux}

Nous posons pour ces verbes la même question : avec les verbes pronominaux, plus précisément dans les quatre formes de pronominal ouvertes à alternance d'auxiliaire, et avec les verbes admettant une alternance de participe, le corpus met-il en évidence des préférences de esse avec la forme courte, et de avè avec la forme longue, ou d'ailleurs toute autre préférence ? Le tableau ci-dessous synthétise les résultats : 


\begin{tabular}{|l|l|l|l|l|}
\hline & \multicolumn{2}{|l|}{$\begin{array}{l}\text { Verbes } \\
\text { pronominaux } \\
\text { avec forme } \\
\text { longue }\end{array}$} & \multicolumn{2}{l|}{$\begin{array}{l}\text { Verbes } \\
\text { pronominaux } \\
\text { avec forme } \\
\text { courte }\end{array}$} \\
\cline { 2 - 6 } & esse & avè & esse & avè \\
\hline $\begin{array}{l}\text { asciuvassi ((s')essuyer, } \\
\text { s'assécher) }\end{array}$ & & 1 & & \\
\hline buscassi ((s')attraper, obtenir) & & & & 4 \\
\hline cumprassi ((s')acheter) & 1 & & & 3 \\
\hline francassi (réchapper de) & 3 & 2 & 1 & 3 \\
\hline liccassi (recevoir) & & & 1 & \\
\hline pinsassi (penser, se dire) & 1 & & 1 & 1 \\
\hline $\begin{array}{l}\text { sciaccassi (se goinfrer de, ou } \\
\text { verbe support) }\end{array}$ & 2 & 1 & & 11 \\
\hline tuccassi (se & & & & \\
toucher ; percevoir) & 2 & & & \\
\hline Totaux & 9 & 4 & 3 & \\
\hline
\end{tabular}

Tableau 2 : Mise en relation des alternances d'auxiliaire et des alternances de participes au sein des verbes pronominaux.

Huit verbes pronominaux relevant des quatre formes pronominales ouvertes à alternance d'auxiliaire que sont le réfléchi à objet inaliénable, l'éthique à objet aliénable avec un verbe bivalent, le réciproque avec objet grammatical et le pronominal avec un verbe monovalent inergatif), et admettant deux participes ${ }^{31}$, ont été relevés dans les corpus, totalisant 27 occurrences.

Difficile de tirer des conclusions à partir de chiffres si bas, mais si on les accepte à titre indicatif on voit que le tableau ci-dessus ne corrobore pas l'hypothèse selon laquelle il y aurait une relation privilégiée entre d'un côté auxiliaire esse et participe court, et de l'autre auxiliaire avè et participe long (même avec le verbe pinsà cité dans la grammaire de Franchi). C'est même dans ces deux zones que se trouvent les chiffres les plus bas (4 et 3 supra). Les totaux sembleraient donc même indiquer plutôt le contraire, c'est-à-dire une association plus fréquente entre auxiliaire esse et participe long ( 9 des 12 esse), et auxiliaire avè et participe court (11 des 15 avè).

31 Respectivement asciuvatu et asciuttu, buscatu et buscu, cumpratu et compru, francatu et francu, liccatu et leccu, pinsatu et pensu, sciaccatu et sciaccu, tuccatu et toccu. 
En réalité, ce qui nous semble pertinent est l'existence de productions sur la totalité des quatre cas de figure. Il n'y a pas d'automatisme car toutes les associations sont envisageables, comme le montre la répartition des résultats sur les quatre colonnes dont aucune n'est vide. Les relations entre les deux variables ne sont donc pas mécaniques. Mais le choix à l'intérieur de chaque variable n'est pas pour autant aléatoire : chaque domaine de variation a sa propre explication, sans qu'il y ait de corrélation possible entre les deux explications. Ce sont deux facteurs indépendants, chacun avec sa logique. C'est la raison pour laquelle on se trouve face à toute la gamme des combinaisons de facteurs, en fonction des intentions signifiantes.

\section{Explications en l'absence de corrélation entre les deux variations}

Quelle réponse alors apporter à la question initialement posée, qui était celle en corse d'une éventuelle corrélation entre la variation d'auxiliaire et la variation de participe passé ? La réponse diffère selon qu'on considère les verbes simples ou les verbes pronominaux.

Dans le cas des verbes simples et en termes qualitatifs, les verbes qui varient leur auxiliaire sont des intransitifs à même de pondérer leurs composantes But et Source :

Des intransitifs à même de sélectionner avè quand 1) leur sujet est Source et But animé et agentif, et esse quand leur sujet est Source et But inanimé (corre, saltà, vulà, principià, cumincià), ou 2) qui sélectionnent avè quand leur sujet est Source et But animé et volitif, et esse quand leur sujet est Source et But animé mais non volitif (cambià, parturì), ou 3) qui sélectionnent avè quand leur sujet est animé Source et But partiellement contrôleur, et esse quand leur sujet est animé Source et But mais non contrôleur (sculiscià, sguilli).

En termes quantitatifs, sont concernés au moins 350 verbes attestés selon nos comptages, ce qui est peu face à la masse de tous les autres verbes simples monovalents et des verbes simples bivalents, qui prennent avè, mais beaucoup par rapport à d'autres langues romanes (une petite trentaine de monovalents en français) et bien plus que l'italien qui avec une petite centaine de verbes est considéré comme la référence dans ce domaine (Abeille \& Godard : 446, Legendre \& Sorace : 211).

Quant aux verbes simples qui varient leur participe ce sont, à 4 exceptions près ${ }^{32}$ auxquelles s'ajoute cumincià qui accepte les deux valences, des verbes bivalents, et souvent transitifs avec un objet subissant un changement d'état. Les intransitifs ne sont quasiment pas concernés par ce phénomène.

Ce sont aussi des verbes qui appartiennent au $1^{\mathrm{er}}$ groupe, le plus productif ${ }^{33}$, à 4 exceptions près : piove, qui appartient au $2^{\mathrm{e}}$ groupe, valè / valoir au $4^{\mathrm{e}}$, et apparì et scumparì au 3 e . À noter que trois de ces quatre verbes étaient déjà des exceptions en termes de valence.

32 Qui sont des intransitifs inaccusatifs : piove (pleuvoir), tuccà (toucher, devoir), apparì (apparaître) et scumparì (disparaître).

33 On distingue en corse 4 groupes : $1^{\text {er }}$ groupe en $-\grave{a}$, avec accentuation arhizotonique et participe en $-a t u .2^{\mathrm{e}}$ groupe : en $-e$ ou $-a$ non-accentué avec accentuation rhizotonique et participe majoritairement en $-u t u .3^{\mathrm{e}}$ groupe : en $-i$ accentué (oxytonique) avec participe en $-i t u$. Et un $4^{\mathrm{e}}$ groupe pour les verbes arhizotoniques en -è accentué, irréguliers et dont 
Sont concernés au moins 150 verbes selon nos comptages, mais outre que l'absence d'une forme dans des corpus ne signe pas son inexistence, n'ont pas été pris en compte les nombreux verbes dont la variation de participe nous a semblé limitée à une région, comme par exemple creda (croire) dont le participe cridutu n'alterne avec crettu que dans le Sud. Casta par exemple (2003 : 102) signale dans le Celavu Mizana la très grande fréquence des formes courtes, par exemple pacu / pigliu pour respectivement les verbes pacà / piglià (payer / prendre), inusitées dans d'autres régions mais compris car le mode de formation des participes courts est inscrit dans la compétence des locuteurs.

Si on considère les verbes simples qui connaissent à la fois la variation d'auxiliaire et celle de participe, il est clair que la monovalence nécessaire à la première et la bivalence typique de la seconde ne prédisposait pas à une large intersection, et de fait on a vu qu'un seul verbe au plus (cumincià) était concerné.

Dans ces conditions quelle réponse apporter à la question d'une éventuelle corrélation entre la variation d'auxiliaire et la variation de participe passé au sein des verbes simples ? Que la question ne se pose quasiment pas, cette langue ayant dissocié les complexités liées aux variations en les cloisonnant autant que faire se peut sur la base de deux groupes distincts.

Dans le cas des verbes pronominaux le tableau est différent : les verbes qui varient leur auxiliaire sont des verbes bivalents, avec objet instancié (voire objet interne dans le cas de l'inergatif), la plupart à même de jouer sur une orientation sujet ou objet. Leur nombre est difficile à évaluer mais élevé, ne serait-ce qu'au travers du pronominal éthique à objet aliénable avec un verbe bivalent (un des quatre pronominaux ouverts à variation), qui admet quasiment tout verbe bivalent, qu'il soit transitif avec sujet contrôleur ou pas, comme respectivement illustré par 44 et 45 ci-dessous :

(44c) Chì pudarà vincia u surrisu quandu a morti s'hè strappatu u pezzu ch'edda bramava ?

(44f) Lorsque la mort a arraché le morceau qu'elle désirait, que pourra vaincre le sourire? (AT), mot à mot Lorsque la mort s'est arraché le morceau qu'elle désirait...

(45c) L'omi si sò scurdati quessa a virità.

(45f) Les hommes ont oublié cette vérité (PP), mot à mot Les hommes se sont oublié cette la vérité.

Quant aux verbes pronominaux qui varient leur participe, ce sont à peu près les mêmes que les verbes bivalents majoritairement $\mathrm{du} 1^{\mathrm{er}}$ groupe déjà mentionnés, auxquels s'ajoutent des verbes simples monovalents qui en emploi pronominal acquièrent un argument supplémentaire (sur le statut du pronom clitique cf. Giancarli 2011), tel campassi (éprouver du bien-être) qui connaît les participes campatu et campu alors que le monovalent nonpronominal campà (vivre) se limite à la seule forme longue.

Si on considère les verbes pronominaux qui connaissent à la fois la variation d'auxiliaire et celle de participe, 8 ont été relevés dans nos corpus, ce qui est une réalisation bien plus modeste que ce que nous anticipions (mais gardons à l'esprit que 7 des 11 types de pronominaux ne prennent que esse et ne sont donc pas ouverts à variation d'auxiliaire), et avec des productions attestées sur les quatre combinaisons possibles (esse + court, esse +

la liste est close : avè, pudè, vulè, valè (valoir), duvè, sapè, parè (sembler), au participe en $-u t u$. 
long, avè + court, avè + long). Le fait que les résultats soient répartis sur la totalité des combinaisons possibles est pour nous le signe qu'il ne faut pas chercher des liens mécaniques entre variables mais plutôt voir des associations à partir des invariants de chaque auxiliaire à l'intérieur du micro-système des auxiliaires, et à partir des invariants de chaque forme participiale à l'intérieur du micro-système des participes. Mais les paramètres thématiques du premier et aspectuo-modaux du second ne sont pas liés, et ne peuvent pas l'être. Rappelons ces invariants, et voyons leur mise en pratique.

Nous donnons à la sélection d'auxiliaire l'explication suivante : avè est sélectionné quand le sujet a le macro-rôle de terme Source, esse est sélectionné quand le sujet correspond à la fois à un terme Source et un terme But, que ce double statut soit le cas de façon intrinsèque (verbes simples) ou acquis par le biais d'une co-référence (verbes pronominaux).

Nous donnons au choix de participe l'explication suivante, basée sur une double relation : la forme courte, fondamentalement adjectivale, présente entre une zone et son complémentaire une Frontière sans épaisseur qui garantit l'atteinte d'un état résultant, alors que la forme longue mobilise directement un domaine notionnel comprenant une Frontière avec épaisseur (intervalle dilaté, et symbolisé par l'infixe -AT- d'ailleurs dans la syllabe sous accent) qui marque un allongement de la phase de transition en repoussant la borne de droite de l'intervalle. Un phénomène de symbolisme phonétique sur la base more sound more time (plus il y a de phonèmes et plus il y a d'occurrences de t) n'est pas exclu.

Cette opposition de nature aspectuelle donne lieu, en présence d'une opération de visée, à des interprétations modales orientées à l'inverse l'une de l'autre : dans le cas de la forme longue il y a échec de validation grâce à une orientation inverse entre la valeur visée par le sujet grammatical et le préconstruit de l'énonciateur; dans le cas de la forme courte il y a atteinte de validation grâce à une orientation à l'identique entre valeur visée par le sujet grammatical et le préconstruit de l'énonciateur.

A titre d'illustration considérons l'extrait suivant au parfait tiré d'un album d'Astérix traduit en corse par Francesca Albertini et qui comprend deux occurrences de pronominal en succession :

(46c) - Ma ùn capisci micca, o Obelix ? Un legiunariu si hà purtatu u nostru Idefix !!! - S'HÀ PURTATU À IDEFIX? L'AGHJU DA FÀ VEDE EIU, PURTÀ SI À IDEFIX, PER TOUTATIS !!!

- Stà bassu, o Obelix, stà bassu !

- MA CHI TANTU CALMA TI È STA BASSU ! VOGLIU U MIO IDEFIX !

(46f) - Mais tu ne comprends pas Obélix ? Un légionnaire a emporté notre Idéfix !!! - EMPORTÉ IDÉFIX? JE VAIS LEUR APPRENDRE, MOI, A EMPORTER IDÉFIX, PAR TOUTATIS !!!

- Du calme, Obélix ! Du calme !

- JE NE VEUX PAS DE CALME ! JE VEUX MON IDÉFIX !!! (RG)

En tant que verbe pronominal éthique à objet aliénable, purtassi est ouvert aux deux auxiliaires. Esse était en effet sélectionnable, dans la mesure où il y a dans ce pronominal un clitique et un sujet grammatical co-référents, avec un clitique si terme Source (au sens d'agent), un objet terme But (u nostru Idefix au sens de patient), et un sujet à la fois terme But (un legiunariu dans sa variante bénéficiaire / détrimentaire) de façon intrinsèque et terme Source de par sa co-référence avec le clitique. L'argument bénéficiaire potentiel du procès et 
l'argument source du procès étant en relation de co-référence, leur identification permet de les assimiler à un actant unique permettant à un legiunariu de réaliser un cumul : le sujet a un double statut Source (par co-référence) + But (au sens de bénéficiaire). Glose : si procure $u$ nostru Idefix pour un legiunariu, avec un legiunariu en relation d'identification avec si.

Esse était sélectionnable, mais n'a pas été sélectionné. Pour la raison que l'orientation ne se fait pas vers le sujet mais vers l'objet, qui est thématique. Peu importe que ce soit un légionnaire qui ait kidnappé Idéfix, le sujet n'est pas affecté d'une perte définitive mais engagé dans la seule récupération de l'objet (cf. l'exclamative vogliu u mio Idefix! / je veux mon Idéfix !!!), d'où in fine la sélection de l'auxiliaire avè. On est même en présence de deux mentions du même verbe avec deux fois l'auxiliaire avè, la première en si hà et l'autre en $s$ 'hà, forme allégée due à la reprise.

Le verbe purtassi dans ce genre de pronominal permet une variation d'auxiliaire, mais pas de participe : en effet purtatu est la seule possibilité, car certes portu existe mais il est le participe d'un autre verbe, non pas purtà mais porghje (tendre, présenter), si bien que portu ne peut en aucun cas commuter avec purtatu. On peut cependant procéder à une manipulation, consistant à remplacer purtassi, qui n'a qu'un seul participe passé, par un verbe synonyme qui en possède deux, en l'occurrence chjappassi (attraper) qui permet chjappatu et chjappu. La manipulation est d'autant plus intéressante que l'extrait comprend deux occurrences de parfait à la suite.

À fin de vérification, et pour ne pas travailler sur nos propres intuitions, nous avons posé la question à la traductrice officielle des albums d'Astérix en langue corse Francesca Albertini, pour voir quels seraient ses choix d'auxiliaire et de participe si elle employait le verbe chjappassi. Les questions que nous nous posions (mais que nous ne lui avons pas formulées) étaient : choisirait-elle le parfait à forme courte ou à forme longue ? Pour les deux mentions du verbe ou pour une seule, et alors laquelle ? Nous lui avons donc présenté, avec l'auxiliaire avè qui était son choix initial justifié ci-dessus, les quatre versions possibles de variation du participe (chjappatu / chjappu, chjappu / chjappatu, chjappatu / chjappatu, сhјарри / chјарри) :

a/ - Un legiunariu si hà chjappatu u nostru Idefix !!! - S'HÀ CHJAPPU À IDEFIX ?

b/ - Un legiunariu si hà chjappu u nostru Idefix !!!

- S'HÀ CHJAPPATU À IDEFIX ?

c/ - Un legiunariu si hà chjappatu u nostru Idefix !!!

- S'HÀ CHJAPPATU À IDEFIX ?

$\mathrm{d} / \quad$ - Un legiunariu si hà chjappu u nostru Idefix !!! - S'HÀ CHJAPPU À IDEFIX ?

La première fait suivre forme longue et forme courte, la deuxième à l'inverse forme courte et forme longue, la troisième se limite à la longue et la dernière à la courte. Sa réponse a été : la séquence en b/, c'est-à-dire qu'elle fait alterner le participe, et ce dans l'ordre où c'est d'abord la forme courte chjappu qui est employée puis la longue chjappatu. Autrement dit elle exploiterait la latitude qui lui est permise par les verbes à doubles participes, en choisissant un participe à un endroit et l'autre participe à l'autre endroit, là où en français on aurait bien sûr une répétition stricte. 
Notre explication s'appuiera sur le contexte et la prise en compte de la spécificité du passage : les deux occurrences de chappassi au parfait se conjuguent avec l'auxiliaire avè, pour les raisons données précédemment à savoir que ce qui prime est la récupération de l'objet. Mais il s'agit d'un dialogue, avec des tours de parole et des sources énonciatives qui alternent. Ici bien qu'il s'agisse des même relations prédicatives il importe de relever que ce ne sont plus les même locuteurs, ni les même relations que chaque locuteur entretient avec l'objet.

Dans la première mention avec le parfait en avè est employé le participe court (si hà chjappu [...]), afin de montrer le résultat en situation tel qu'il est et dont le locuteur (Astérix) prend acte, sans dramatisation ni modalisation supplémentaires, d'autant qu'Astérix sait qu'Idéfix victime du kidnapping est le meilleur ami d'Obélix, inutile d'en rajouter.

Par contre la deuxième mention du verbe chappà se fait avec le participe long $\left(S^{\prime} H \grave{A}\right.$ CHJAPPATU [...]) afin de montrer la difficulté d'intégration par le nouveau locuteur (Obélix) de la réalité, vécue comme problématique. Le nouveau locuteur refuse cette réalité, il aurait voulu qu'il n'en fût pas ainsi, et fait appel à une forme modalisée, relevant plus précisément d'une modalité appréciative (type 3 dans la TOPE) porteuse d'une valuation du procès.

Ce choix est bien sûr à mettre en relation d'une part avec la typographie et d'autre part avec l'accusatif prépositionnel choisis :

- La typographie en lettres capitales exclusivement sur la mention comprenant le participe long et par le second locuteur ( $\left.S^{\prime} H \grave{A} C H J A P P A T U[\ldots]\right)$ veut rendre à l'écrit ce qui à l'oral serait un volume plus élevé (et une plage intonative plus haute) traduisant l'expression d'une forte charge émotionnelle par ce personnage, ce qui confirme sa difficulté à intégrer une information jugée excessivement perturbante.

- En parfaite cohérence avec la typographie sur ce segment va le choix d'un accusatif prépositionnel exclusivement dans la version où le verbe est porteur du participe long et exclusivement dans la question formulée par le second locuteur (S'H IDEFIX ?) mais pas dans l'assertion qui la précède immédiatement (si hà chjappu $\emptyset$ u nostru Idefix !!!) car la question est une reformulation porteuse d'une mise en cause de la véracité d'une relation que l'énonciateur a du mal à intégrer, autrement dit pour ce dernier Idéfix ne peut pas (dans cette relation) jouer le rôle d'objet. On retrouve là la même logique que dans l'emploi de ce marqueur devant l'objet unu dans l'exemple 24 de participe absolu Tuccatu ad unu, toccu u populu tuttu quantu.

\section{Conclusion}

Nous avons vu que, concernant les verbes pronominaux, la variation d'auxiliaire et la variation de participe donnent lieu à toute la gamme des choix possibles, mais les paramètres thématiques à la base de la première et ceux aspectuo-modaux à la base de la seconde ne sont pas liés, ils sont simplement associés dans telle ou telle paire en fonction des intentions signifiantes.

Concernant les verbes simples, l'intersection entre le groupe des verbes à variation d'auxiliaire (monovalent) et celui des verbes à variation de participe (typiquement bivalent) 
est quasiment vide. Ils varient mais chacun de son côté, sans que l'un interagisse avec l'autre. Ce sont deux poches de variation circonscrites à deux sous-classes, d'une part sans doute relativement importantes en particulier au regard d'autres langues romanes mais tout de même largement minoritaires, et d'autre part presque totalement disjointes. La situation d'une complexité maximale avec croisement des variables n'est pas exploitée par la langue.

En fait l'extension de chacune de ces deux variations est soumise à des contraintes :

1) La variation d'auxiliaire entre esse et avè, pour ce qui concerne les verbes monovalents, se voit imposer une limitation de esse au profit de avè. Que ce soit en raison de facteurs internes peut-être spécifiques ${ }^{34}$, ou/et à cause de l'influence du français sur certains verbes (piove / pleuvoir), ou/et en raison d'une évolution générale ${ }^{35}$ affectant également d'autres langues notamment romanes ${ }^{36}$ et qui dans une vision scalaire affecterait les verbes d'abord les moins inaccusatifs ${ }^{37}$.

2) De son côté la variation de participe se voit imposée une limitation de la forme courte au profit de la longue.

- Par exemple certains verbes, même du $1^{\mathrm{er}}$ groupe, n'ont pas deux participes mais un seul (sous forme longue) dans la mesure où la forme courte est déjà le participe unique et long d'un autre verbe ${ }^{38}$, ou parce qu'elle est seulement un adjectif et un adjectif de sens relativement éloigné du verbe ${ }^{39}$.

- La forme courte a parfois un sens supplémentaire autre qu'adjectival / participial, ce qui pourrait la rendre ambiguë (ou changer totalement le sens de la phrase) et conduire les locuteurs à l'éviter ${ }^{40}$.

34 La fragilité de esse se manifeste en corse par la capacité de avè à conjuguer un verbe normalement limité à esse en cas d'ellipse du verbe ou d'éloignement de celui-ci ou de coordination de celui-ci avec un verbe sélectionnant avè (cf. Giancarli 2011 : 143 sqq). "From the perspective of the history of Romance languages we have a continuum: some languages have completed the process of eliminating E [auxiliaire essif]" (Legendre 2007 : 26). Cf. aussi Sorace 2000 : 860, Legendre \& Sorace 2003 : 195, Sorace 2004 : 258.

L'espagnol et le français, mais aussi l'anglais, le suédois ou le norvégien.

37 Cf. l'Auxiliary Selection Hierarchy, Sorace 2004: 258, également appelée Split Intransitivity Hierarchy, Sorace 2011, voir Giancarli 2015 pour une vision critique.

38 Ainsi capità (se produire) permet capitatu mais pas capitu car ce dernier est déjà le participe de capi (comprendre), ou purtà permet purtatu mais pas portu qui est déjà le participe de porghje (tendre).

39 Ainsi ficcà, qui signifie enfoncer ou tromper, admet comme participe ficcatu mais pas ficcu, qui est un adjectif signifiant fatigué.

40 Ainsi salvu et francu, formes courtes associées respectivement à salvà (sauver) et francà (franchir), peuvent aussi être des prépositions au sens de sauf. De ce fait seule la forme longue est possible dans Francatu à San Martinu, a ghjenti t'hà in càrciara, in magazenu, in casetta o caseddu, a so roba da l'invernu è da u branu (ASA) au sens de Après la Saint Martin les gens ont dans la cave, le grenier, la maisonnette ou la cabane des champs, leurs provisions pour l'hiver et le printemps. Sélectionner francu à San Martinu au lieu de 
- Enfin il s'agit peut-être là aussi d'une évolution générale dans laquelle la forme courte serait dans un parfait une construction ancienne, archaïsante, et en perte de vitesse. L'italien antique a connu des parfaits à forme courte (Laurent : 163, Tekavcic : 437-438) mais ne les connaît plus guère, et ils ont existé en vieux provençal et en vieux catalan (De Dardel : 77). Ils existaient d'ailleurs déjà en latin, puisque le latin tardif comptait des doublets tel pour le verbe audere (oser) ausatu / ausu (Laurent : 355-356) aussi bien que le latin classique pour par exemple potare (boire) potatu / potu (id., p. 61), si ce n'est qu'en latin le participe initial (court) et son correspondant long n'étaient pas liés exactement au même verbe mais à deux verbes partiellement distincts, celui dont le participe était long étant un verbe intensif / itératif dérivé du verbe de base dont pour sa part le participe était court. Par exemple sur canere au participe court cantum a été formé un intensif itératif (cantare) au participe cantatum, long. De même accipere au participe court acceptus a donné acceptare au participe long acceptatus (De Dardel : 80-81), cf. Note 10.

Il existe donc des contraintes qui concourent à brider l'extension de l'une et de l'autre variation. Et ces contraintes vont sans doute dans le sens d'une évolution de fond. A partir de là on peut avancer une double prévision :

- Au sein des intransitifs : si la variation à l'intérieur des deux groupes (ceux des verbes acceptant esse et avè, et ceux des verbes acceptant forme longue et forme courte) va en se rétrécissant, les chances d'intersection entre ces deux groupes ne peuvent que diminuer d'autant, mais rappelons que l'intersection est déjà réduite à sa portion congrue. Ces deux groupes de variation minoritaires et largement sans intersection sont appelés 1) à devenir encore plus minoritaires, 2) à réduire leur part d'intersection et donc à s'éloigner de plus en plus l'un de l'autre.

- Au sein des pronominaux, la variation d'auxiliaire est toujours bien vivante, mais la variation de participe diminue puisque à l'intérieur du groupe des verbes acceptant l'alternance entre forme longue et forme courte cette dernière est en décroissance. La zone d'intersection entre les deux variations ne peut donc également que se réduire, même si les invariants à l'intérieur des deux zones de variations restent intacts.

\section{Références}

Abeille, A. ; Godard, D. (2002). The Syntactic Structure of French Auxiliaries, Language 78, $3: 404-452$.

Amaral, P. ; Howe, C. (2013). Nominal and Verbal Plurality in the Diachrony of the Portuguese Present Perfect, Verbal Plurality and Distributivity, Cabredo-Hofherr \& Laca (ed.), Linguistische Arbeiten 546, De Gruyter : 25-54.

Blatt, F. (1952). Précis de syntaxe latine, Lyon : IAC.

Cabredo-Hofherr, P. ; Laca B. ; Carvalho S. De (2010). When Perfect means Plural : the Present Perfect in North-Eastern Brazilian Portuguese, in : Cabredo Hofherr \& Laca (ed.) Layers of Aspect, Stanford, C : CSLI, 67-100.

Casta, S. (2003). La syntaxe du corse, Gap : CRDP de Corse.

francatu à San Martinu aurait remplacé un participe absolu au sens de une fois franchie la Saint-Martin (après la Saint-Martin) par un Groupe Prépositionnel à sens exceptif (excepté à la Saint-Martin). 
Ceccaldi, M. (1974). Dictionnaire corse-français, Limoges : Klincksieck.

Culioli, A. (1986). La frontière, Cahiers Charles V 8 : 161-170.

Culioli, A. (1990). Pour une linguistique de l'énonciation, tome 1, Gap : Ophrys.

Culioli, A. ; Culioli, G.-D. ; Culioli, G.-S. ; Culioli, V.-S. (1998). Dizziunariu Corsu-Francese, Ajaccio : DCL Editions.

De Dardel, R. (1962). La fonction des participes romans sans suffixe, Cahiers Ferdinand de Saussure, 19, Droz, Genève : 77-86.

Durand, O. (2003). La lingua corsa, Brescia : Paideia Editrice.

Franchi, G.-G. (2000). Puntelli di gramatica, Literatura corsa, 3, Ajaccio : CRDP de Corse.

Giacomo-Marcellesi, M. (1988). Histoire du corse, Lexicon der Romanischen Linguistik, vol. 4, Tübingen : Max Niemeyer Verlag : 820-829.

Giancarli, P.-D. (2011). Les Auxiliaires ÊTRE et AVOIR : étude comparée corse, français, acadien et anglais, Rennes : PUR, collection «Rivages Linguistiques ».

Giancarli, P.-D. (2014). L'accusatif prépositionnel en corse, Faits de Langues 43, Peter Lang, Berne : 197-212.

Giancarli, P.-D. (2015). Auxiliary Selection with Intransitive and Reflexive verbs: the Limits of Gradience, Followed by a Proposal, Auxiliary Selection Revisited: Gradience and Gradualness, R. Kailuweit \& M. Rosemeyer (ed.), FRIAS book series "Linguae \& Littera", vol. 44, De Gruyter, Berlin/Boston : 79-120

Giorgi, A. ; Panesi F. (1997). Tense and Aspect: from Semantics to Morphosyntax, Nex York : Oxford University Press.

Hale, K. ; Keyser S. (2002). Prolegomenon to a Theory of Argument Structure, London : MIT Press.

Herslund, M. (1996). Partitivité et possession inaliénable, Faits de Langue 7, La relation d'appartenance : 33-42.

Laurent, R. (1999). Past Participles from Latin to Romance, Linguistics vol. 133, University of California Press.

Legendre, G. (2007). On the Typology of Auxiliary Selection, Lingua, 117-9 : 1522-1540.

Legendre, G. ; Sorace A. (2003). Auxiliaires et intransitivité en français et dans les langues romanes, Les Langues Romanes, Sciences du Langage, CNRS Éditions : 185-233.

Newman, P. (2012). Pluractional Verbs: an Overview, Verbal Plurality and Distributivity, Cabredo-Hofherr \& Laca (ed.), Linguistische Arbeiten 546, De Gruyter : 185-210.

Riegel, M. ; Pellat J.-C. ; Rioul R. (1994) [1997]. Grammaire méthodique du français, Paris : PUF.

Rohlfs, G. (1968). Grammatica storica della lingua italiana e dei suoi dialetti, Torino, Einaudi editore, vol. 2.

Salvi, G. (1982). Sulla storia sintattica della costruzione romanza habeo + participio, Revue Romane 17 : 118-133.

Salvi, G. (1987). Syntactic Restructuring in the Evolution of Romance Auxiliaries, Historical Development of Auxiliaries, M. Harris ; P. Ramat (ed.), Mouton de Gruyter : 225-236.

Sorace, A. (2000). Gradients in Auxiliary Selection with Intransitive Verbs, Language 76, 4 : 859-890.

Sorace, A. (2004). Gradience at the Lexicon-Syntax Interface: Evidence from Auxiliary Selection and Implications for Unaccusativity, in : A. Alexiadou, M. Everaert and E. Anagnostopoulou, (ed.), The Unaccusativity Puzzle, Oxford University Press : 243-268.

Sorace, A. (2011). Gradience in Split Intransitivity: the End of the Unaccusativity Hypothesis? Archivio Glottologico Italiano 96 : 67-86.

Tekavcic, P. (1972). Grammatica storica dell'italiano, Bologna, Il Mulino. 
Vincent, N. (1982). The Development of the Auxiliaries habere et esse in Romance, in: N. Vincent ; M. Harris, (ed.), Studies in the Romance Verb, London / Canberra, Croom Helm :71-96.

\section{Sources du corpus et leurs abréviations}

Antoni, P. (2000). Detti è fatti - Paroles et actions, Ajaccio : La Marge, édition bilingue, traduction du corse en français par l'auteur. Roman. (DF)

Antoni, P. (2002). L'ultimu paciaghju / Le dernier diseur de paix, Ajaccio : La Marge, édition bilingue, traduction du corse en français par l'auteur. Roman. (UP)

Beckett, S. [1948] (1988). En Attendant Godot, Paris : Les Éditions de Minuit.

Traduction en corse de Geronimi D. (1985). Intantu, in Rigiru 23, Ajaccio : Éditions Cyrnos et Méditerrané. Pièce de théâtre. (EAG)

Biancarelli, M. (2003). 51 Pegasi astru virtuali / 51 Pégase, Astre virtuel, Ajaccio, Albiana, édition bilingue, traduction en français par J. Ferrari. Roman. (PAV)

Chateaubriand, F.-R. de (1802). Génie $d u$ Christianisme, http://un2sg4.unige.ch/athena/chateaubriand/chat_ren.html.

Traduction en corse (extrait) de Studii Corsi di Tilò (1985). La Lettre de la Corse, numéro spécial bilinguisme, 4. Roman. (G)

Comiti, J.-M. (1998). Da una sponda à l'altra, recueil de nouvelles trilingues corse-françaisitalien, Ajaccio : éd. A. Piazzola, Centre Culturel Universitaire, traduction du corse en français par l'auteur. Roman. (DS)

Coti, R. (2001). A Travisagna, http://www.interromania.com/documents/travisagna-739.html.

Traduction en français par Multedo, R. (2001). Nouvelle. (AT)

Coti, R. [1978] (2004). Intornu à l'essezza, Casa di u Populu Corsu, Paris : éditions Éolienne.

Traduction en français par Dalmas-Alfonsi, P. (2004). Essai. (IE)

Coti, R. (2002-2008). U Taravu, éditoriaux du Journal mensuel de la région du Taravu, Biguglia.

Les traductions dans le sens corse-français ont été faites par l'auteur, Coti, N. ; Bruschini, L. ou Jureczek. M. Journal. (UT)

Coti, R. (2009). A Sant'Andria, ville d'Ajaccio et Collectivité Territoriale de Corse.

Traduction en français par Stefanelli, M. Spectacle musical et théâtral. (ASA)

Daudet, A. [1869] (2001). Lettres de mon moulin, in Euvres, tome III, Paris : Bibliothèque de la Pléiade, Gallimard.

Traduction en corse de Ceccaldi, M. (1980). Lettare da u me mulinu, Paris : Klincksieck. Recueil de contes. (LDM)

Fischer, N. (1999). Les lettres de Toussainte / E lettere di Santa, Ajaccio : DCL Éditions, édition bilingue, traduction en corse de Rocchi, C. Correspondance. (LT)

Franchi, G.-G. (1988). E fole di zia Anna-Maria, Ajaccio : Éditions A. Piazzola, traduction en français de Franchi, G.-G. Contes. (F)

Giacomo-Marcellesi, M. (1989). Contra Salvatica, Aix-en-Provence : Edisud, édition bilingue, traduction en français de Giacomo-Marcellesi, M. Légendes et contes. (CS)

Goscinny, R. ; Uderzo A. (2003). Astérix et la rentrée gauloise, Paris : Éditions Albert René.

Traduction en corse de Albertini, F. (2004). Asterix gira è volta... à a scola, Paris : Éditions Albert René. Bande dessinée. (RG)

Saint-Exupery, A. de [1943] (1990). Le Petit Prince, Paris : Gallimard.

Traduction en corse de Casta, S. (1990). U Principellu, Ajaccio : Akenaton \& Squadra di u Finusellu. Conte. (PP) 
Simonpoli, P. (sous la direction de) (1995). La chasse en Corse, Gênes : Parc Naturel Régional de Corse. Textes originaux en corse, traductions en français par Casta, S. ; Notini, G. ; Dalmas-Alfonsi, P. ; Franchi, G.-G. ; De Zerbi, G. ; Fusina, G. Recueil d'articles thématiques. (CEC)

Thiers, J. (1996). A barca di a Madonna, Ajaccio : Albiana.

Traduction en français de Bonerandi, H. (1997). La vierge à la barque, Ajaccio : Albiana. Roman. (ABM)

Thiers, J. (1990). A Funtana d'Altea, Ajaccio : Albiana.

Traduction en français par l'auteur (1992). Les glycines d'Altea, Ajaccio : Albiana. Roman. (AFA)

Thiers, J. (2003). In corpu à Bastia, Ajaccio : Albiana.

Traduction en français de Thiers, A.-L. (2004). Le ventre de Bastia, Ajaccio : Albiana. Roman (ICB) 